\title{
Interpretation of searches for supersymmetry with simplified models
}

\author{
S. Chatrchyan et al. ${ }^{*}$ \\ (CMS Collaboration)
}

(Received 10 January 2013; published 23 September 2013)

\begin{abstract}
The results of searches for supersymmetry by the CMS experiment are interpreted in the framework of simplified models. The results are based on data corresponding to an integrated luminosity of 4.73 to $4.98 \mathrm{fb}^{-1}$. The data were collected at the LHC in proton-proton collisions at a center-of-mass energy of $7 \mathrm{TeV}$. This paper describes the method of interpretation and provides upper limits on the product of the production cross section and branching fraction as a function of new particle masses for a number of simplified models. These limits and the corresponding experimental acceptance calculations can be used to constrain other theoretical models and to compare different supersymmetry-inspired analyses.
\end{abstract}

DOI: 10.1103/PhysRevD.88.052017

PACS numbers: 14.80.Ly, 12.60.Jv, 13.85.Rm

\section{INTRODUCTION}

The results of searches for supersymmetry (SUSY) [1] at particle colliders are often used to test the validity of a few, specific, theoretical models. These models predict a large number of experimental observables at hadron colliders as a function of a few theoretical parameters. Most of the SUSY analyses performed by the Compact Muon Solenoid (CMS) experiment present their results as an exclusion of a range of parameters for the constrained minimal supersymmetric standard model (CMSSM) [2-4]. However, the results of the SUSY analyses can be used to test a wide range of alternative models, since many SUSY and non-SUSY models predict a similar phenomenology. These similarities inspired the formulation of the simplified model framework for presenting experimental results [5-9]. Specific applications of these ideas have appeared in Refs. [10,11].

A simplified model is defined by a set of hypothetical particles and a sequence of their production and decay. For each simplified model, values for the product of the experimental acceptance and efficiency $(\mathcal{A} \times \epsilon)$ are calculated to translate a number of signal events into a signal cross section. From this information, a 95\% confidence level upper limit (UL) on the product of the cross section and branching fraction $\left([\sigma \times \mathcal{B}]_{\mathrm{UL}}\right)$ is derived as a function of particle masses. The simplified model framework can quantify the dependence of an experimental limit on the particle spectrum or a particular sequence of particle production and decay in a manner that is more general than the CMSSM. Furthermore, the values of $[\sigma \times \mathcal{B}]_{\mathrm{UL}}$ can be compared with theoretical predictions from a SUSY or non-SUSY model to determine whether the theory is compatible with data.

*Full author list given at the end of the article.

Published by the American Physical Society under the terms of the Creative Commons Attribution 3.0 License. Further distribution of this work must maintain attribution to the author(s) and the published article's title, journal citation, and DOI.
This paper collects and describes simplified model interpretations of a large number of SUSY-inspired analyses performed on data collected by the CMS Collaboration in 2011 [12-26]. The simplified model framework was also applied by CMS to a limited number of analyses in 2010 [27]. The ATLAS Collaboration has published similar interpretations [28-34].

The paper is organized as follows. Section II provides a brief description of the CMS analyses considered here; Sec. III describes simplified models; Sec. IV demonstrates the calculation of the product of the experimental acceptance and efficiency and the upper limits on cross sections; Sec. V contains comparisons of the results for different simplified models and analyses; Sec. VI contains a summary.

\section{THE CMS DETECTOR AND ANALYSES}

The CMS detector consists of a silicon tracker, an electromagnetic calorimeter, and a hadronic calorimeter, all located within the field volume of a central solenoid magnet, and a muon-detection system located outside the magnet [35]. Information from these components is combined to define objects such as electrons, muons, photons, jets, jets identified as $b$ jets ( $b$-tagged jets), and missing transverse energy $\left(\mathscr{E}_{\mathrm{T}}\right)$. The exact definition of these objects depends on the specific analysis, and can be found in the analysis references. The data were collected by the CMS experiment at the Large Hadron Collider in proton-proton collisions at a center-of-mass energy of $7 \mathrm{TeV}$. Unless stated otherwise, the data corresponds to an integrated luminosity of $4.98 \pm 0.11 \mathrm{fb}^{-1}$ [36].

The descriptions of the analyses are categorized by the main features of the event selection. Detailed descriptions of these analyses can be found in Refs. [12-26]. The target of these analyses is a signal of the production of new, heavy particles that decay into standard model particles and stable, neutral particles that escape detection. The stable, neutral particles can produce a signature of large $\ddot{E}_{\mathrm{T}}$. The standard model also produces $\not{E}_{\mathrm{T}}$ in top quark, weak gauge 
boson, and heavy flavor production. Fluctuations in energy deposition in the detector can also produce significant $\mathscr{E}_{\mathrm{T}}$ in quantum chromodynamics processes.

\section{A. All-hadronic}

Events contain two or more high transverse momentum $\left(p_{\mathrm{T}}\right)$ jets and significant $\mathscr{E}_{\mathrm{T}}$. Events with isolated leptons are rejected to reduce backgrounds from $t \bar{t}, W$, and $Z$ boson production. A selection on kinematic discriminants is applied to reduce backgrounds containing $\mathbb{E}_{\mathrm{T}}$. The names of the discriminants label the analyses: $\alpha_{\mathrm{T}}[12], \mathrm{H}_{\mathrm{T}}+$ jets [13], and $M_{\mathrm{T} 2}$ [14]. The $\alpha_{\mathrm{T}}$ [37] and $M_{\mathrm{T} 2}$ [38,39] variables are both motivated by the kinematics of new particle pair production and decay into two visible systems of jets and a pair of invisible particles. The $\mathrm{H}_{\mathrm{T}}+$ jets analysis, instead, uses a selection on the negative vector sum $\left(\mathrm{H}_{\mathrm{T}}\right)$ and the scalar sum $\left(H_{\mathrm{T}}\right)$ of the transverse momentum of each jet. The $M_{\mathrm{T} 2}$ analysis uses data corresponding to an integrated luminosity of $4.73 \pm 0.10 \mathrm{fb}^{-1}$.

The $\alpha_{\mathrm{T}}$ analysis mentioned above also categorizes events with one, two, or at least three jets that satisfy a $b$-tagging requirement. The $M_{\mathrm{T} 2} b$ analysis modifies the $M_{\mathrm{T} 2}$ selection mentioned above and requires at least one $b$-tagged jet. The $\ddot{E}_{\mathrm{T}}+b$ analysis [15] follows a similar strategy as the $\mathrm{H}_{\mathrm{T}}+$ jets analysis, but selects events with one, two, or at least three $b$-tagged jets.

\section{B. Single lepton + jets}

Events are selected with one high- $p_{\mathrm{T}}$, isolated lepton (electron or muon), jets, and significant $\not{E}_{\mathrm{T}}$. Three analyses are considered in this paper [17]. The lepton spectrum $(e / \mu \mathrm{LS})$ and lepton projection $(e / \mu \mathrm{LP})$ methods exploit the expected correlation between the lepton $p_{\mathrm{T}}$ and $\mathbb{E}_{\mathrm{T}}$ from $W$ boson decays to separate a potential signal from the main backgrounds of $t \bar{t}$ and $W+$ jets production. The artificial neutral network (ANN) method applies an ANN that is based on event properties (jet multiplicity, $H_{\mathrm{T}}$, transverse mass, and the azimuthal angular separation between the two highest- $p_{\mathrm{T}}$ jets) to separate backgrounds from the expectations of a CMSSM benchmark model.

Two other analyses [18] require also two or more $b$-tagged jets. In the first analysis $\left(e / \mu \geq 2 b+\not \mathbb{E}_{\mathrm{T}}\right)$, the $W^{+}, W^{-}$, and $t \bar{t}$ background distributions from simulation are corrected to match the measured $\mathscr{E}_{\mathrm{T}}$ spectrum at low $H_{\mathrm{T}}$, and then the corrected prediction is extrapolated to high $H_{\mathrm{T}}$ and high $\mathbb{E}_{\mathrm{T}}$. A selection on $\ddot{E}_{\mathrm{T}}$ significance $\left(\mathrm{Y}_{\mathrm{MET}}\right)$ and $H_{\mathrm{T}}$ is used in the second analysis $(e / \mu \geq$ $\left.3 b, \mathrm{Y}_{\mathrm{MET}}\right)$. The $\mathrm{Y}_{\mathrm{MET}}$ variable is defined as the ratio of $\mathbb{E}_{\mathrm{T}}$ to $\sqrt{H_{\mathrm{T}}}$.

\section{Opposite-sign dileptons}

Events are selected with two leptons (electrons or muons) having electric charge of the opposite sign (OS), jets, and significant $\not{E}_{\mathrm{T}}$. In one (OS $\left.e / \mu+\not_{\mathrm{T}}\right)$ analysis
[21], a signal is defined as an excess of events at large values of $\boldsymbol{E}_{\mathrm{T}}$ and $H_{\mathrm{T}}$. In a second (OS $e / \mu$ edge) analysis [21], a search is performed for a characteristic kinematic edge in the dilepton mass distribution $m_{\ell^{+} \ell^{-}}$. In these two analyses events with an $e^{+} e^{-}$or $\mu^{+} \mu^{-}$pair with invariant mass of the dilepton system between $76 \mathrm{GeV}$ and $106 \mathrm{GeV}$ or below $12 \mathrm{GeV}$ are removed in order to suppress $Z / \gamma^{*}$ events, as well as low-mass dilepton resonances. A third analysis (OS $e / \mu$ ANN) [22] applies a selection on the output of an ANN that is based on seven kinematic variables constructed from leptons and jets, to discriminate the signal events from the background.

Two other complementary analyses focus directly on the two leptons from $Z$-boson decay by applying an invariant mass selection [25]. With this requirement, the main source of $\mathbb{E}_{\mathrm{T}}$ arises from fluctuations in the measurement of jet energy. One analysis $\left(Z+\mathbb{E}_{\mathrm{T}}\right)$ determines this background from a control sample that differs only in the presence of a $Z$ boson. A second analysis (JZB) applies a kinematic variable denoted $\mathrm{JZB}$, which is defined as the difference between the sum of the vector elements of the $p_{\mathrm{T}}$ of the jets and the $p_{\mathrm{T}}$ of the boson candidate.

The last analysis in this group selects events consistent with a $W$ boson decaying to jets produced in association with a $Z$ boson decaying to leptons, and searches for an excess of events in the $\mathscr{E}_{\mathrm{T}}$ distribution. This analysis is part of the combined lepton (comb. leptons) analysis [24] that targets a signal containing gauge boson pairs and $\not_{\mathrm{T}}$ : $W Z$, $Z Z+\mathbb{E}_{\mathrm{T}}$.

\section{Same-sign dileptons}

Events are selected with two leptons (electrons or muons) having electric charge of the same sign (SS), and significant $\not{E}_{\mathrm{T}}$. One analysis (SS $e / \mu$ ) uses several different selections on $\mathscr{E}_{\mathrm{T}}$ and $H_{\mathrm{T}}$ to suppress backgrounds [19]. A second analysis $(\mathrm{SS}+b)$ requires at least one $b$-tagged jet [20]. A third analysis makes no requirements on jet activity. It limits backgrounds by applying more stringent lepton identification criteria. Results from this analysis are included in the combined lepton results [24].

\section{E. Multileptons}

Events are selected containing at least three leptons. Selections are made on the values of several event variables, including $\mathbb{E}_{\mathrm{T}}, H_{\mathrm{T}}$, and the invariant mass of lepton pairs [23,24]. One analysis applies a veto on $b$-tagged jets to remove most of the $t \bar{t}$ background, and is included in the combined lepton results [24].

\section{F. Photons}

Events are selected containing one photon, two jets, and $\mathbb{E}_{\mathrm{T}}\left(\gamma \mathrm{jj}+\mathbb{E}_{\mathrm{T}}\right)$, or two photons, one jet, and $\mathbb{E}_{\mathrm{T}}\left(\gamma \gamma \mathrm{j}+\mathbb{E}_{\mathrm{T}}\right)$ [26]. The requirement of a photon and $\mathscr{E}_{\mathrm{T}}$ is sufficient to remove most backgrounds. 


\section{G. Inclusive}

The razor analysis integrates several event categories [16]. Events are required to contain jets and zero, one, or two leptons (electrons and muons) with a further classification based on the presence of a $b$-tagged jet. The razor variable [40] is a ratio of a jet system mass to a transverse mass. The distribution in the razor variable is highly correlated with the mass values of new particles for hypothesized signals but skewed to relatively smaller values for backgrounds. Values of the razor variable are chosen to reduce backgrounds while accepting signal events in a similar manner as for the $\alpha_{\mathrm{T}}$ and $M_{\mathrm{T} 2}$ analyses. The razor analysis uses data corresponding to an integrated luminosity of $4.73 \pm 0.10 \mathrm{fb}^{-1}$.

\section{SIMPLIFIED MODELS}

A simplified model is defined by a set of hypothetical particles and a sequence of their production and decay. In this paper, the selection of models is motivated by the particles and interactions of the CMSSM or models with generalized gauge mediation [41]. For convenience, the particle naming convention of the CMSSM is adopted, but none of the specific assumptions of the CMSSM are imposed. The CMSSM assumptions include relationships among the new particle masses, their production cross sections and distributions, and their decay modes and distributions. In the simplified models under consideration, only the production process for two primary particles is considered. Each primary particle can undergo a direct decay or a cascade decay through an intermediate new particle. Each particle decay chain ends with a neutral, undetected particle, denoted LSP (lightest supersymmetric particle) in text and $\tilde{\chi}_{\text {LSP }}$ in equations. $\tilde{\chi}_{\text {LSP }}$ can represent a neutralino or gravitino LSP. The masses of the primary particle and the LSP are free parameters. When the model includes the cascade decay of a mother particle (mother) to an intermediate particle (int), the mass of the intermediate particle depends on $m_{\text {mother }}, m_{\mathrm{LSP}}$, and a parameter $x$, according to the equation $m_{\text {int }}=x m_{\text {mother }}+(1-x) m_{\mathrm{LSP}}$. The value of $x$ can be anywhere in the range from zero to one, but values of $x=\frac{1}{4}, \frac{1}{2}$ and $\frac{3}{4}$ are used here.

TABLE I. Summary of the simplified models used in the interpretation of results.

\begin{tabular}{|c|c|c|c|c|}
\hline Model name & Production mode & Decay & Visibility & References \\
\hline$\overline{\mathrm{T} 1}$ & $\bar{g} \tilde{g}$ & $\tilde{g} \rightarrow q \bar{q} \tilde{\chi}_{\mathrm{LSP}}$ & All-hadronic & {$[12-14,16]$} \\
\hline $\mathrm{T} 2$ & $\tilde{q} \tilde{q}^{*}$ & $\tilde{q} \rightarrow q \tilde{\chi}_{\mathrm{LSP}}$ & All-hadronic & {$[12,13,16]$} \\
\hline \multirow[t]{3}{*}{$\mathrm{T} 5 \mathrm{zz}$} & \multirow[t]{3}{*}{$\tilde{g} \tilde{g}$} & \multirow[t]{3}{*}{$\tilde{g} \rightarrow q \bar{q} \tilde{\chi}_{2}^{0}, \tilde{\chi}_{2}^{0} \rightarrow Z \tilde{\chi}_{\mathrm{LSP}}$} & All-hadronic & {$[13,14]$} \\
\hline & & & Opposite-sign dileptons & {$[25]$} \\
\hline & & & Multileptons & [23] \\
\hline \multirow[t]{2}{*}{$\mathrm{T} 3 \mathrm{w}$} & \multirow[t]{2}{*}{$\tilde{g} \tilde{g}$} & $\tilde{g} \rightarrow q \bar{q} \tilde{\chi}_{\mathrm{LSP}}$ & \multirow[t]{2}{*}{ Single lepton + jets } & \multirow[t]{2}{*}{ [17] } \\
\hline & & $\tilde{g} \rightarrow q \bar{q} \tilde{\chi}_{1}^{ \pm}, \tilde{\chi}_{1}^{ \pm} \rightarrow W^{ \pm} \tilde{\chi}_{\mathrm{LSP}}$ & & \\
\hline T5lnu & $\tilde{g} \tilde{g}$ & $\tilde{g} \rightarrow q \bar{q} \tilde{\chi}_{1}^{ \pm}, \tilde{\chi}_{1}^{ \pm} \rightarrow \ell \nu \tilde{\chi}_{\mathrm{LSP}}$ & Same-sign dileptons & [19] \\
\hline $\mathrm{T} 31 \mathrm{~h}$ & $\tilde{g} \tilde{g}$ & $\begin{array}{c}\tilde{g} \rightarrow q \bar{q} \tilde{\chi}_{\mathrm{LSP}} \\
\tilde{g} \rightarrow q \bar{q} \tilde{\chi}_{2}^{0}, \tilde{\chi}_{2}^{0} \rightarrow \ell^{+} \ell^{-} \tilde{\chi}_{\mathrm{LSP}}\end{array}$ & Opposite-sign dileptons & {$[21,22]$} \\
\hline T1bbbb & $\tilde{g} \tilde{g}$ & $\tilde{g} \rightarrow b \bar{b} \tilde{\chi}_{\mathrm{LSP}}$ & All-hadronic (b) & {$[12,14-16]$} \\
\hline \multirow[t]{4}{*}{$\mathrm{T} 1 \mathrm{tttt}$} & \multirow[t]{4}{*}{$\tilde{g} \tilde{g}$} & \multirow[t]{4}{*}{$\tilde{g} \rightarrow t \bar{t} \tilde{\chi}_{\mathrm{LSP}}$} & All-hadronic (b) & {$[12,14,15]$} \\
\hline & & & Single lepton + jets $(b)$ & [18] \\
\hline & & & Same-sign dileptons (b) & {$[19,20]$} \\
\hline & & & Inclusive (b) & [16] \\
\hline $\mathrm{T} 2 \mathrm{bb}$ & $\tilde{b} \tilde{b}^{*}$ & $\tilde{b} \rightarrow b \tilde{\chi}_{\mathrm{LSP}}$ & All-hadronic (b) & {$[12,16]$} \\
\hline T6ttww & $\tilde{b} \tilde{b}^{*}$ & $\tilde{b} \rightarrow t \tilde{\chi}^{-}, \tilde{\chi}^{-} \rightarrow W^{-} \tilde{\chi}_{\mathrm{LSP}}$ & Same-sign dileptons (b) & [20] \\
\hline $\mathrm{T} 2 \mathrm{tt}$ & $\tilde{t}^{*}$ & $\tilde{t} \rightarrow t \tilde{\chi}_{\mathrm{LSP}}$ & All-hadronic (b) & {$[12,16]$} \\
\hline TChiSlepSlep & $\tilde{\chi}_{1}^{ \pm} \tilde{\chi}_{2}^{0}$ & $\begin{array}{c}\tilde{\chi}_{2}^{0} \rightarrow \ell^{ \pm} \tilde{\ell}^{\mp}, \tilde{\ell} \rightarrow \ell \tilde{\chi}_{\mathrm{LSP}} \\
\tilde{\chi}_{1}^{ \pm} \rightarrow \nu \tilde{\ell}^{ \pm}, \tilde{\ell}^{ \pm} \rightarrow \ell^{ \pm} \tilde{\chi}_{\mathrm{LSP}}\end{array}$ & Multileptons & {$[23,24]$} \\
\hline TChiwz & $\tilde{\chi}_{1}^{ \pm} \tilde{\chi}_{2}^{0}$ & $\tilde{\chi}_{1}^{ \pm} \rightarrow W^{ \pm} \tilde{\chi}_{\mathrm{LSP}}, \tilde{\chi}_{2}^{0} \rightarrow Z \tilde{\chi}_{\mathrm{LSP}}$ & Multileptons & {$[23,24]$} \\
\hline TChizz & $\tilde{\chi}_{2}^{0} \tilde{\chi}_{3}^{0}$ & $\tilde{\chi}_{2}^{0}, \tilde{\chi}_{3}^{0} \rightarrow Z \tilde{\chi}_{\mathrm{LSP}}$ & Multileptons & {$[23,24]$} \\
\hline T5gg & $\tilde{g} \tilde{g}$ & $\tilde{g} \rightarrow q \bar{q} \tilde{\chi}_{2}^{0}, \tilde{\chi}_{2}^{0} \rightarrow \gamma \tilde{\chi}_{\mathrm{LSP}}$ & Photons & [26] \\
\hline T5wg & $\tilde{g} \tilde{g}$ & $\begin{array}{c}\tilde{g} \rightarrow q \bar{q} \tilde{\chi}_{2}^{0}, \tilde{\chi}_{2}^{0} \rightarrow \gamma \tilde{\chi}_{\mathrm{LSP}} \\
\tilde{g} \rightarrow q \bar{q} \tilde{\chi}_{1}^{ \pm}, \tilde{\chi}_{1}^{ \pm} \rightarrow W^{ \pm} \tilde{\chi}_{\mathrm{LSP}}\end{array}$ & Photons & [26] \\
\hline
\end{tabular}


The simplified models with a T1, T3, and T5 prefix are all models of gluino pair production, with different assumptions about the gluino decay. Those with a T2 and T6 prefix are models of squark-antisquark production, with different assumptions on the type of squark or the pattern of squark decay. Those with a TChi prefix are models of chargino and neutralino production and decay. In the simplified models under consideration, the $W$ and $Z$ bosons decay to any allowed final state. A detailed description of the specific models follows. Table I provides a summary.

\section{A. T1, T1bbbb, T1tttt}

The T1 model is a simplified version of gluino pair production. Each gluino undergoes a three-body decay to a light-flavor quark-antiquark pair and the LSP $\left(\tilde{g} \rightarrow q \bar{q} \tilde{\chi}_{\text {LSP }}\right)$. Ignoring the effects of additional radiation and jet reconstruction, this choice produces a final state of four jets $+\not E_{\mathrm{T}}$. The T1bbbb and T1tttt models are modifications of the T1 model in which the gluino decays exclusively into $b$ or $t$ quark-antiquark pairs. After accounting for the unobserved LSPs, the kinematic properties of events from the T1tttt model are indistinguishable by the analyses considered here to those from alternative simplified models, such as gluino decay to a top squark and an antitop quark, followed by top squark decay to a top quark and the LSP $\left(\tilde{g} \rightarrow t \tilde{t}^{*}, \tilde{t}^{*} \rightarrow \bar{t} \tilde{\chi}_{\text {LSP }}\right.$ ) [20]. For simplicity, only the T1ttt model is considered.

\section{B. T2, T2bb, T2tt, T6ttww}

The T2 model is a simplified version of squarkantisquark production. Each squark undergoes a twobody decay to a light-flavor quark and the LSP $\left(\tilde{q} \rightarrow q \tilde{\chi}_{\text {LSP }}\right)$. Ignoring the effects of additional radiation and jet reconstruction, this choice produces a final state of two jets $+\mathscr{E}_{\mathrm{T}}$. The T2bb and T2tt models are versions of bottom and top squark production, respectively, with the bottom (top) squark decaying to a bottom (top) quark and the LSP. The T6ttww model is a version of direct bottom squark production, with the bottom squark decaying to a top quark, a $W$ boson, and the LSP.

\section{T3w, T3lh}

The models with the T3 prefix are also based on gluino pair production. One gluino has a direct decay to a light-flavor quark-antiquark pair and the LSP, as in the T1 model. The other gluino has a cascade decay through an intermediate particle, denoted as $\tilde{\chi}_{2}^{0}$ or $\tilde{\chi}_{1}^{ \pm}$. In the T3w model, the cascade decay is a two-body decay of the chargino to a $W$ boson and the LSP. For the T3lh model, the cascade decay is a three-body decay of a heavy neutralino to a lepton pair and the $\operatorname{LSP}\left(\tilde{\chi}_{2}^{0} \rightarrow \ell^{+} \ell^{-} \tilde{\chi}_{\text {LSP }}\right)$. If a heavy neutralino $\tilde{\chi}_{2}^{0}$ decays to the LSP $\tilde{\chi}_{\text {LSP }}$ and a pair of leptons, the edge occurs at $m_{\ell^{+} \ell^{-}}=m_{\tilde{\chi}_{2}^{0}}-m_{\tilde{\chi}_{\text {LSP }}}$, corresponding to the region of kinematic phase space where $\tilde{\chi}_{\text {LSP }}$ is produced at rest in the $\tilde{\chi}_{2}^{0}$ rest frame.

\section{T5Inu, T5zz}

The models with T5 prefix are also based on gluino pair production. Both gluinos undergo cascade decays. The T5lnu model has each gluino decay to a quarkantiquark pair and chargino that undergoes a three-body decay to a lepton, neutrino, and LSP. The decay can produce SS dileptons, due to the Majorana nature of the gluino. The T5zz model has each gluino decay to a quark-antiquark pair and an intermediate neutralino that undergoes a two-body decay to a $Z$ boson and the LSP. When both $Z$ bosons in an event decay to a quarkantiquark pair, and ignoring the effects of additional radiation and jet reconstruction, the T5zz model produces a final state of eight jets $+\mathbb{E}_{\mathrm{T}}$.

\section{E. TChiSlepSlep, TChiwz, TChizz}

These models are simplified versions of the direct production and decay of charginos and neutralinos or neutralino pairs. The TChiSlepSlep and TChiwz models are versions of chargino-neutralino production. The former has neutralino and chargino cascade decays through a charged slepton to three electrons, muons, and taus in equal rate, while the latter has direct decays to gauge bosons and LSPs. The TChiSlepSlep model does not include the decay $\tilde{\chi}_{2}^{0} \rightarrow \tilde{\nu} \nu$, since this will not produce a multilepton signature. The TChizz model, instead, is a version of neutralino pair production and decay into $Z$ bosons.

\section{F. T5gg, T5wg}

The T5gg model is a version of gluino pair production in which each gluino decays to a quark-antiquark pair and an intermediate neutralino, which further decays to a photon and a massless LSP. The T5wg model, instead, has one gluino decaying to quark-antiquark pair and an intermediate neutralino that decays to a photon and the LSP, and the second gluino decaying to a quark-antiquark pair and a chargino that decays to a $W$ boson and the LSP. The neutralino and chargino masses are set to a common value to allow an interpretation in models of gauge mediation. The intermediate neutralino is labeled as the next-to-LSP (NLSP).

The calculation of $\mathcal{A} \times \epsilon$ for each simplified model uses the PYTHIA [42] event generator with the SUSY differential cross sections for gluino, squark-antisquark, and neutralino and chargino pair production. The decays of non-Standard Model particles are performed with a constant amplitude, so that no spin correlations exist between the decay products. The primary particle masses are varied between $100 \mathrm{GeV}$ and $1500 \mathrm{GeV}$. The theoretical prediction for the production cross section is not needed to calculate $[\sigma \times \mathcal{B}]_{\mathrm{UL}}$. However, it is informative to compare the values of $[\sigma \times \mathcal{B}]_{\mathrm{UL}}$ with the production cross section expected in a benchmark model. The selected benchmark is the CMSSM cross section prediction for 
gluino pair, squark-antisquark, or neutralino and chargino pair production. The cross sections are determined at next-to-leading order $\left(\sigma^{\mathrm{NLO}}\right)$ accuracy for gaugino pair production, and at NLO with next-to-leading-logarithmic contributions $\left(\sigma^{\mathrm{NLO}+\mathrm{NLL}}\right)$ for the other processes [43-48]. In both the calculation of particle production and the reference cross sections, extraneous SUSY particles are decoupled. For example, the contributions from squarks are effectively removed by setting the squark mass to a very large value when calculating the acceptance and cross section for gluino pair production. The cross sections are presented under the assumption of unit branching ratios, even for models such as T3w that consider two different decay modes of the gluino.

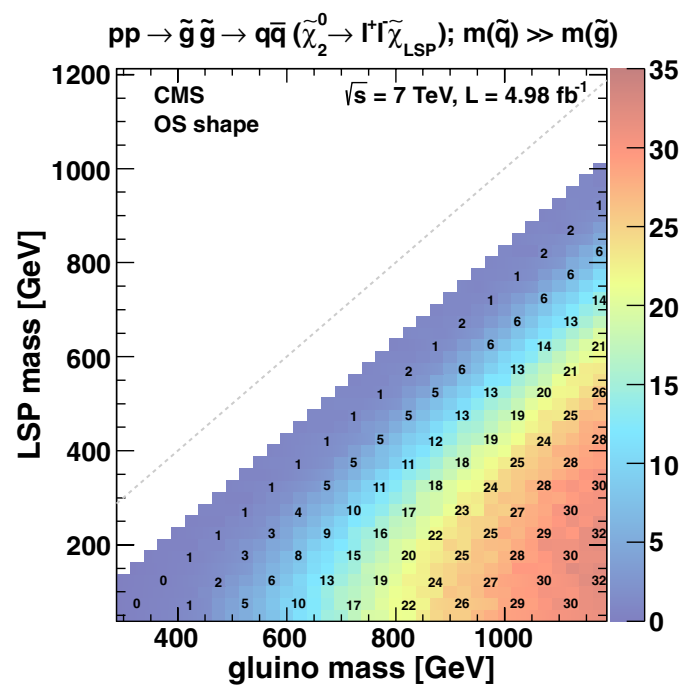

$\mathrm{m}\left(\tilde{\chi}_{2}^{0}\right)=0.5 \mathrm{~m}\left(\tilde{\chi}_{L S P}\right)+0.5 \mathrm{~m}(\tilde{\mathbf{g}})$

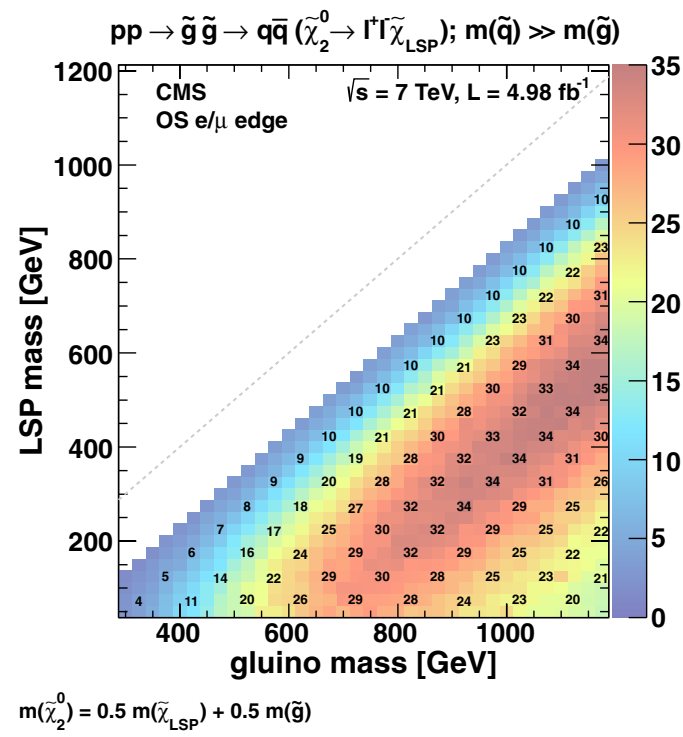

\section{LIMIT SETTING PROCEDURE}

The method used to set exclusion limits is common to all simplified models and analyses. In this section, the procedure is presented using the interpretation of two different OS dilepton analyses [21] as an example.

The reference simplified model is T31h, which can yield pairs of OS leptons not arising from $Z$-boson decays. The mass of the intermediate neutralino produced in the gluino decay chain is set using $m_{\tilde{\chi}_{2}^{0}}=\frac{1}{2}\left(m_{\tilde{g}}+m_{\tilde{\chi}_{\text {LSP }}}\right)$, corresponding to $x=\frac{1}{2}$. The parameter $x$ influences the patterns of cascade decays. The mass splitting between the gluino and the intermediate particle, $(1-x)\left(m_{\tilde{g}}-m_{\tilde{\chi}_{\text {LSP }}}\right)$, influences the observable hadronic energy, while the mass


FIG. 1 (color online). OS dileptons [21]: Product of the experimental efficiency and acceptance (left) and the upper limit on the product of the cross section and branching fraction (right) for the T3lh model from the $\mathbb{E}_{\mathrm{T}}$ and $H_{\mathrm{T}}$ selection (top) and from edge reconstruction (bottom). Results are shown as a function of gluino and LSP mass, with the intermediate neutralino mass set using $x=0.5$. 


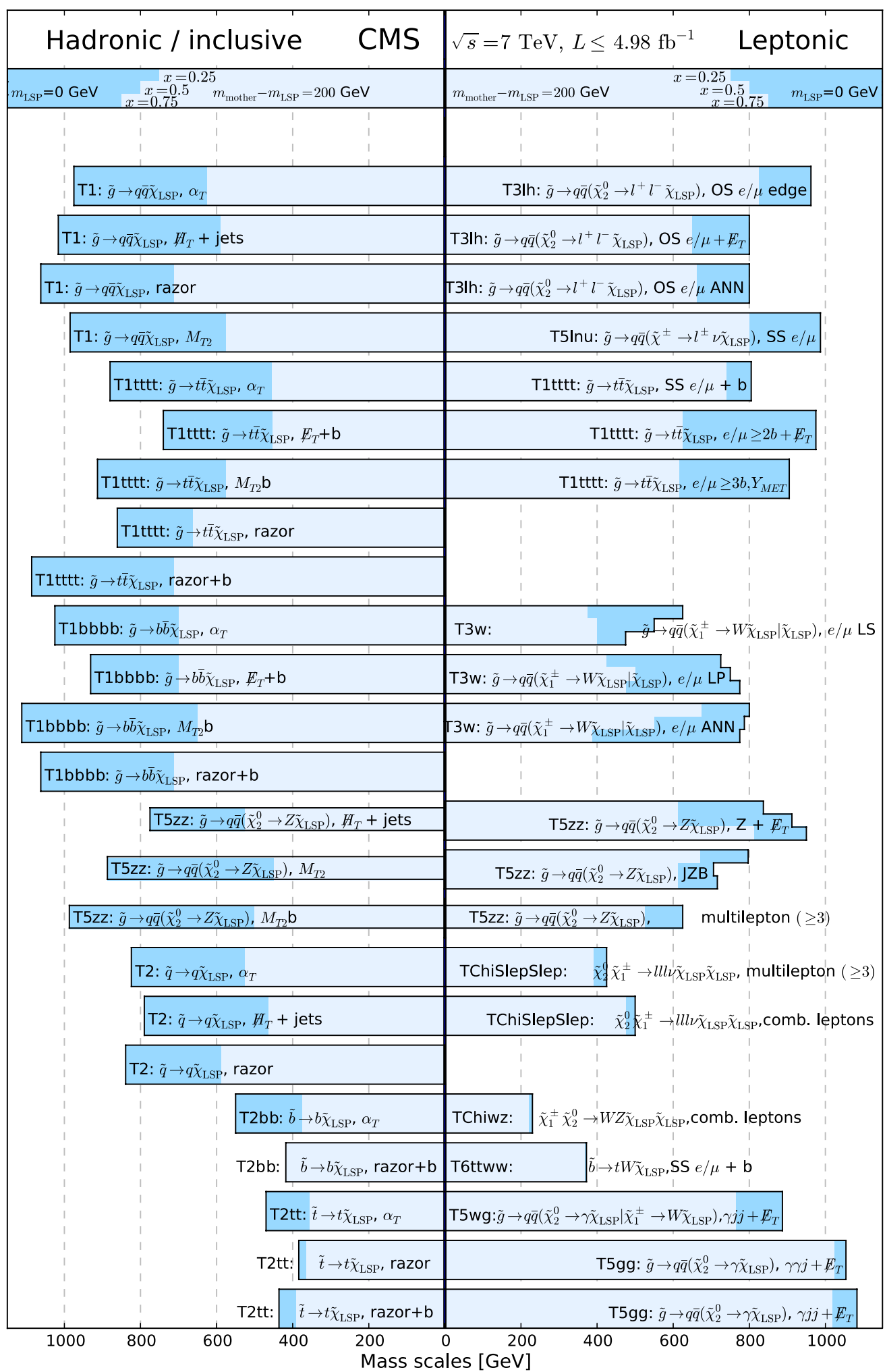

FIG. 2 (color online). Exclusion limits for the masses of the mother particles, for $m_{\mathrm{LSP}}=0 \mathrm{GeV}$ (dark blue) and $m_{\text {mother }}-m_{\mathrm{LSP}}=$ $200 \mathrm{GeV}$ (light blue), for each analysis, for the hadronic and razor results (left) and the leptonic results (right). The limits are derived by comparing the allowed $[\sigma \times \mathcal{B}]_{\mathrm{UL}}$ to the theory described in the text. For the T3, T5, and TChiSlepSlep models, the mass of the intermediate particle is defined by the relation $m_{\mathrm{int}}=x m_{\text {mother }}+(1-x) m_{\mathrm{LSP}}$. For the T3w and T5zz models, the results are presented for $x=0.25,0.5,0.75$, while for the T3lh, T5lnu, and TChiSlepSlep models, $x=0.5$. The lowest mass value for $m_{\text {mother }}$ depends on the particular analysis and the simplified model. 
splitting between the intermediate particle and the LSP, $x\left(m_{\tilde{g}}-m_{\tilde{\chi}_{\text {LSP }}}\right)$, influences the energy of the leptonic decay products or the $\mathbb{E}_{\mathrm{T}}$. For large $x$, the signal is expected to have lower $H_{\mathrm{T}}$ and higher $\mathscr{E}_{\mathrm{T}}$, and possibly higher- $p_{\mathrm{T}}$ leptons. Conversely, for small $x$, the signal should have higher $H_{\mathrm{T}}$ and lower $\ddot{E}_{\mathrm{T}}$, and possibly lower- $p_{\mathrm{T}}$ leptons. Results are shown for a counting experiment based on $H_{\mathrm{T}}$ and $\mathscr{E}_{\mathrm{T}}$ selections (OS $e / \mu+\mathscr{E}_{\mathrm{T}}$ ), and the edge reconstruction in the dilepton invariant mass $\left(m_{\ell^{+} \ell^{-}}\right)$distribution (OS $e / \mu$ edge).

In the first step, the event selection is applied to simulated simplified model events. The ratio of selected to generated events determines $\mathcal{A} \times \epsilon$. The uncertainty on this quantity, which is a necessary input to the limit calculation, is described in the analysis references. This calculation is repeated for different values of the gluino and LSP masses. Values of $\mathcal{A} \times \epsilon$ for the two selections are shown in Fig. 1 (left).

The acceptance of the $\mathbb{E}_{\mathrm{T}}$-based analysis (top left) increases with increasing gluino mass, since the $\mathbb{E}_{\mathrm{T}}$ and gluino mass are correlated. However, this acceptance decreases for smaller gluino-LSP mass splitting. The acceptance of the edge-based analysis (bottom left) is relatively larger for small gluino-LSP mass splitting, but decreases for larger gluino mass. This decrease is an artifact of a choice made in the analysis to limit the $m_{\ell^{+} \ell^{-}}$distribution to $m_{\ell^{+} \ell^{-}}<300 \mathrm{GeV}$. For small mass splitting, the presence of initial-state radiation (ISR) can strongly influence the experimental acceptance. The uncertainty on ISR is difficult to estimate. For this reason, some analyses report results for only a restricted region of mass splittings.

Estimates of $\mathcal{A} \times \epsilon$, the background, and their uncertainties are used to calculate $[\sigma \times \mathcal{B}]_{\mathrm{UL}}$ for the given model using the $\mathrm{CL}_{\mathrm{s}}$ criterion [49,50]. A gluino and LSP mass pair in a simplified model is excluded if the derived $[\sigma \times \mathcal{B}]_{\mathrm{UL}}$ result is below the predicted $\sigma^{\mathrm{NLO}+\mathrm{NLL}}$ for those mass values.

Values of $[\sigma \times \mathcal{B}]_{\mathrm{UL}}$ are shown in Fig. 1 (right) for the two analyses. The edge analysis (bottom right) has less of a stringent selection on $\mathbb{E}_{\mathrm{T}}$ than the counting experiment (top right): for the former the signal regions are defined by $H_{\mathrm{T}}>300 \mathrm{GeV}$ and $\not{E}_{\mathrm{T}}>150 \mathrm{GeV}$, while for the latter different signal regions are obtained requiring high $H_{\mathrm{T}}\left(H_{\mathrm{T}}>600 \mathrm{GeV}\right.$ and $\left.\mathscr{E}_{\mathrm{T}}>200 \mathrm{GeV}\right)$, high $\mathbb{E}_{\mathrm{T}}$ $\left(H_{\mathrm{T}}>300 \mathrm{GeV}\right.$ and $\left.\mathscr{E}_{\mathrm{T}}>275 \mathrm{GeV}\right)$ or tight selection criteria $\left(H_{\mathrm{T}}>600 \mathrm{GeV}\right.$ and $\left.\not_{\mathrm{T}}>275 \mathrm{GeV}\right)$. As a result, the edge analysis sets stronger limits than the counting analysis in this particular topology.

The expected limit and its experimental uncertainty, together with the observed limit and its theoretical uncertainty based on $\sigma^{\mathrm{NLO}+\mathrm{NLL}}$ for gluino pair production, are shown as curves overlaying the exclusion limit. In the case of the OS $e / \mu$ edge analysis, the most stringent limit on the gluino mass is obtained at around $900 \mathrm{GeV}$ for low LSP masses, and for the OS $e / \mu$ analysis it is around $775 \mathrm{GeV}$.
The quoted estimates are determined from the observed exclusion based on the theoretical production cross section minus $1 \sigma$ uncertainty.

The contours of constant $\mathcal{A} \times \epsilon$ do not coincide with those of constant $[\sigma \times \mathcal{B}]_{\mathrm{UL}}$. For the OS $e / \mu+\mathbb{E}_{\mathrm{T}}$, this is an artifact of the changing uncertainty on $\mathcal{A} \times \epsilon$ as a function of gluino and LSP masses, signal contamination, and the observed distribution of $\mathscr{E}_{\mathrm{T}}$. In the OS $e / \mu$ edge analysis, this occurs because the allowed area of the signal $m_{\ell^{+} \ell^{-}}$distribution varies with the mass difference between the gluino and LSP.

\section{RESULTS AND COMPARISONS}

This section presents the results obtained applying the procedure described in Sec. IV to the CMS analyses presented in Sec. II. The individual results are described in detail in the analysis references, but comparisons of the results are presented in this paper for the first time. For each analysis, the lower limit on particle masses in a simplified model is determined by comparing $[\sigma \times \mathcal{B}]_{\mathrm{UL}}$ with the predicted $\sigma^{\mathrm{NLO}+\mathrm{NLL}}$ or $\sigma^{\mathrm{NLO}}$ as described in Sec. III. Only the observed $[\sigma \times \mathcal{B}]_{\mathrm{UL}}$ values are used. The limits are thus subject to statistical fluctuations.

Figure 2 illustrates the results of the hadronic and inclusive analyses (left) and the leptonic analyses (right). Comparisons are made for two reference points of the mother and LSP masses: one with a massless LSP (M0, dark blue in Fig. 2), and one with a fixed mass splitting between the mother particle and the LSP of $200 \mathrm{GeV}$ $(\Delta M 200$, light blue in Fig. 2). The results shown in Fig. 2 are summarized below.

\section{A. All-hadronic}

This class of analyses sets limits on those models, such as T1, T2, and T5zz, that produce several jets, but few leptons. The $\alpha_{\mathrm{T}}$ and $\mathrm{H}_{\mathrm{T}}+$ jets analyses yield similar limits in the T1 and T2 models despite the differences in their event selections. In the case of the T5zz model, the $M_{\mathrm{T} 2}$ analysis is more sensitive to the model's mass splitting than the $\mathrm{H}_{\mathrm{T}}+$ jets analysis: for $M 0$, the $M_{\mathrm{T} 2}$ analysis sets the stronger limit, while for $\Delta M 200$ the $\mathrm{H}_{\mathrm{T}}+$ jets analysis is more sensitive. This is expected, since the $M_{\mathrm{T} 2}$ analysis uses a higher cut on $H_{\mathrm{T}}$ than the $\mathrm{H}_{\mathrm{T}}+$ jets analysis. In general, the limits for the T5zz model are reduced with respect to the $\mathrm{T} 1$ and $\mathrm{T} 2$ models, because of the reduced amount of $\not_{\mathrm{T}}$ in cascade decays.

The $\mathscr{E}_{\mathrm{T}}+b$, the $M_{\mathrm{T} 2} b$, and the $\alpha_{\mathrm{T}}$ analyses set limits on the T1bbbb, T1tttt, T2bb, and T2tt models, visible in Fig. 2 (left). The three analyses set comparable limits for $\Delta M 200$, but the $M_{\mathrm{T} 2} b$ and $\alpha_{\mathrm{T}}$ analyses set the stronger limits for $M 0$. For the T1ttt model, the $M_{\mathrm{T} 2} b$ analysis is most sensitive.

The $M_{\mathrm{T} 2} b$ analysis is also compared with the $M_{\mathrm{T} 2}$ analysis with no $b$-tagging requirement. The limit for the $M_{\mathrm{T} 2} b$ analysis on the T1bbbb model is stronger than for the 
$M_{\mathrm{T} 2}$ analysis on the T1 model, since many of the backgrounds are removed by requiring a $b$-tagged jet, allowing for a lower threshold on the $M_{\mathrm{T} 2}$ variable. Also, the limit on the T5zz model from the $M_{\mathrm{T} 2} b$ analysis is stronger than for the $M_{\mathrm{T} 2}$ analysis, even though the $b$-tagged jets from the T5zz model arise mainly through the decay $Z \rightarrow b \bar{b}$.

\section{B. Single lepton + jets}

This class of analyses is sensitive to simplified models that produce $W$ bosons or direct decays to leptons. The $e / \mu$ LS, LP, and ANN analyses set limits on the T3w model for an intermediate (chargino) mass corresponding to $x=\frac{1}{4}, \frac{1}{2}$, and $\frac{3}{4}$.

The LS and LP analyses are sensitive to the kinematic properties of the $W$ boson produced in the chargino decay. For a large mass splitting between the mother and LSP $(M 0)$, the LP and ANN limits are less sensitive to $x$ than the LS limit. For a fixed mass splitting ( $\Delta M 200)$, however, the ANN limit is more sensitive. The limits for $M 0$ are stronger for all three analyses, with LP and ANN setting the best limits.

The $e / \mu \geq 2 b+\not_{\mathrm{T}}$ and $e / \mu \geq 3 b, \mathrm{Y}_{\mathrm{MET}}$ analyses set limits on the T1tttt model.

\section{Opposite-sign dileptons}

The $\mathrm{Z}+\mathbb{E}_{\mathrm{T}}$ and JZB analyses both set limits on the T5zz model relying on the leptonic decays of one of the $Z$ bosons. The $\mathrm{Z}+\mathbb{E}_{\mathrm{T}}$ analysis sets the stronger limit for $x=\frac{3}{4}$ and $M 0$, for which more $\mathbb{E}_{\mathrm{T}}$ is produced on average. The JZB analysis has the opposite behavior, since the separation between signal and background in the JZB variable is maximized in the signal when the $\mathscr{E}_{\mathrm{T}}$ and $\mathrm{Z}-p_{\mathrm{T}}$ vectors point in the same direction. Therefore, the best limit is set for $x=\frac{1}{4}$.

Limits are also set on the T3lh model, with the nonresonant decay of the intermediate neutralino to leptons, by the $\not_{\mathrm{T}}$, the edge-based, and the neural-networkbased analyses. The edge-based analysis sets significantly stronger limits.

\section{Same-sign dileptons}

The T5lnu model produces equal numbers of OS and SS dileptons. Limits are set on the T5lnu model by the SS dilepton analysis. No comparisons are made for the OS dilepton analyses as these are expected to be much less sensitive due to their larger backgrounds.

The SS dilepton analysis with a $b$-tagged jet is used to set limits on the T1ttt and the T6ttww models. The analysis is not strongly sensitive to mass splittings, and a similar limit is set for the case of $M 0$ or $\Delta M 200$.

\section{E. Multileptons}

Limits are set on the TChiSlepSlep model, which produces leptons through slepton decays but not through gauge boson decays. A limit is set on the chargino mass (which equals the heavy neutralino mass) near $500 \mathrm{GeV}$, which is not strongly dependent on the mass splitting. The limits on the model TChiwz are significantly reduced because of the corresponding reduction from the branching fraction of the gauge bosons into leptons. A limit on the T5zz model is also set. For the $\Delta M 200$ case the limit is competitive with the limits set by the hadronic analyses, despite the low $Z \rightarrow \ell^{+} \ell^{-}$branching fraction.

\section{F. Photons}

Limits are set on the T5gg and T5wg models, which produce two isolated photons and $\not_{\mathrm{T}}$ or one isolated photon and $\mathscr{E}_{\mathrm{T}}$, respectively. The one- and two-photon analyses set comparable limits on the T5gg model. In addition, the one-photon analysis sets a competitive limit on the T5wg model.

\section{G. Inclusive}

The razor and razor $+b$ analyses set limits on the T1, $\mathrm{T} 2$, T1bbbb, T1tttt, T2bb, and T2tt models. The limits on each of these models are comparable with the best limits set by individual, exclusive analyses.

Figure 3 illustrates the best hadronic or leptonic result for each simplified model. Excluding the photon signatures, the best limits for the $M 0$ scenario exclude gluino masses below $1 \mathrm{TeV}$ and squark masses below $800 \mathrm{GeV}$. For the $\Delta M 200$ scenario, the limit is reduced to near $800 \mathrm{GeV}$ and $600 \mathrm{GeV}$, respectively. The limits on the gluino mass from the photon signatures are near $1.1 \mathrm{TeV}$, regardless of the mass splitting.

Figure 4 illustrates the exclusion contours in the twodimensional plane of the mother versus LSP mass for the T1 (T1bbbb), T2 (T2bb), T5zz, T3w, T1ttt, and T5gg (T5wg) models. The results shown in Figs. 2 and 3 are a subset of these results. Regions where the analyses, due to the uncertainty in the acceptance calculation, do not produce a limit are denoted by dashed lines. Figure 4 (upper left) shows the exclusion contours of the T1 and T1bbbb models using the hadronic and $b$-tagged hadronic analyses. This tests the dependence on the assumption of whether the gluino decays to light or heavy flavors. Solid (dashed) lines are used for the T1 (T1bbbb) model. The $\alpha_{\mathrm{T}}$ analysis covers a larger area in the gluino-LSP mass plane for the T1bbbb model than the hadronic decays do for the T1 model. However, this comparison is only valid if the gluino indeed decays only to bottom quarks. The fully hadronic $\mathrm{H}_{\mathrm{T}}+$ jets and $\alpha_{\mathrm{T}}$ analyses cover a similar region, while the $M_{\mathrm{T} 2}$ analysis covers comparatively less. The inclusive analysis is particularly sensitive when the difference in mass between the mother and LSP is small, a situation known as a "compressed spectrum."

Figure 4 (upper right) compares the exclusion contours of the T2 and T2bb models. The $\alpha_{\mathrm{T}}$ and $\mathrm{H}_{\mathrm{T}}+$ jets analyses set similar limits on the T2 model. The $\alpha_{\mathrm{T}}$ analysis sets 


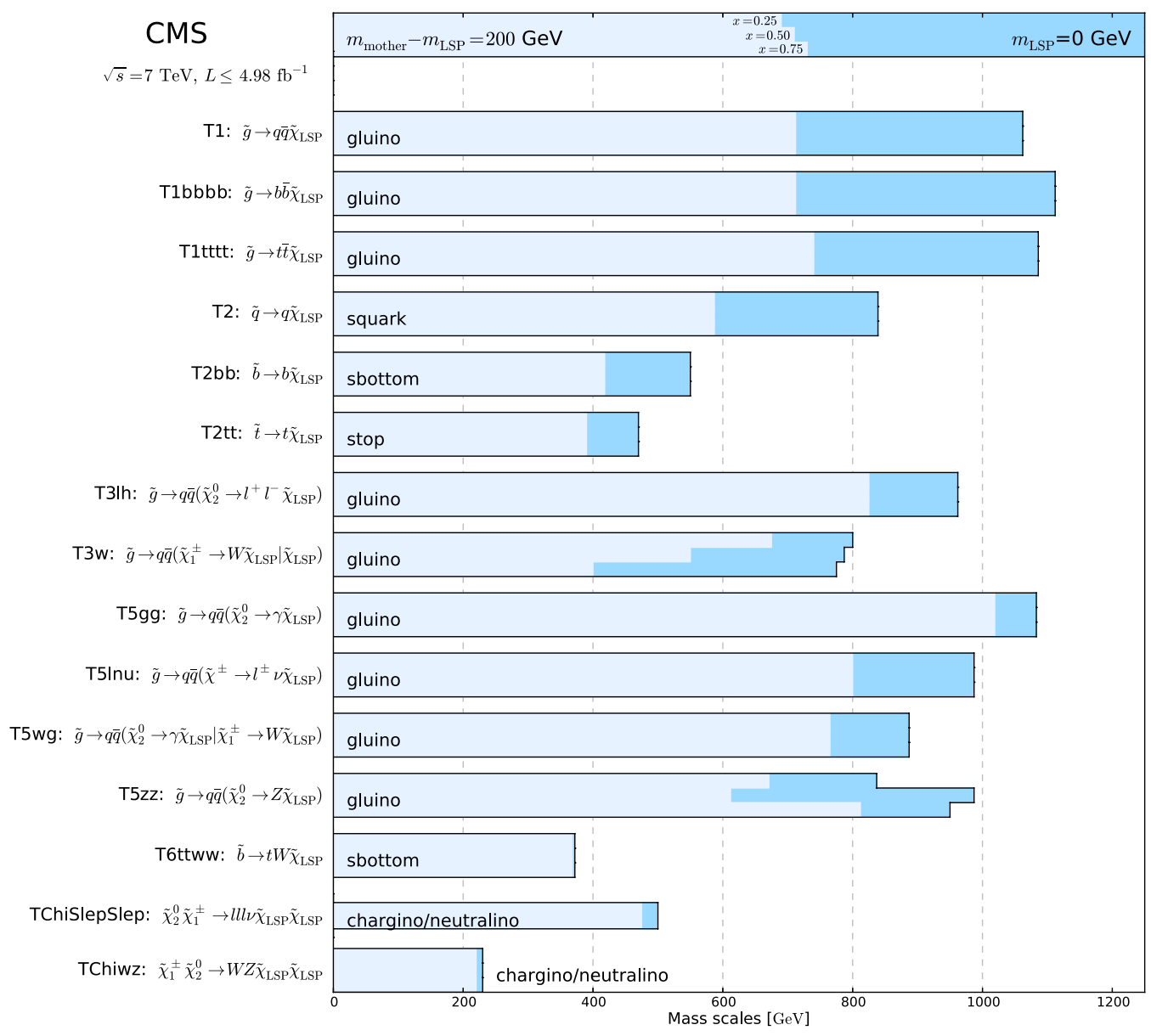

FIG. 3 (color online). Best exclusion limits for the masses of the mother particles, for $m_{\mathrm{LSP}}=0 \mathrm{GeV}$ (dark blue) and $m_{\text {mother }}-$ $m_{\mathrm{LSP}}=200 \mathrm{GeV}$ (light blue), for each simplified model, for all analyses considered. For the T3, T5, and TChiSlepSlep models, the mass of the intermediate particle is defined by the relation $m_{\text {int }}=x m_{\text {mother }}+(1-x) m_{\mathrm{LSP}}$. For the T3w and T5zz models, the results are presented for $x=0.25,0.5,0.75$, while for the T3lh, T5lnu, and TChiSlepSlep models, $x=0.5$. The lowest mass value for $m_{\text {mother }}$ depends on the particular analysis and the simplified model.

weaker limits on the T2bb model, but the reference cross section is a factor of 8 smaller than for the T2 model. The inclusive analysis sets the overall strongest limits, particularly in the low-mass splitting region.

Figure 4 (middle left) compares the exclusion contours of the T5zz model. The T5zz model comparison demonstrates the complementarity of leptonic, hadronic, and $b$-tagged hadronic analyses. In particular, the leptonic analyses are more limiting for smaller mass splittings, while the hadronic analyses are more limiting for larger gluino masses.

Figure 4 (middle right) compares the exclusion contours of the T3w model. The $e / \mu$ ANN and $e / \mu$ LP analyses provide comparable results. The $e / \mu \mathrm{LS}$ spectrum analysis excludes a smaller region.

Figure 4 (bottom left) compares the exclusion contours of the T1tttt model. The inclusive analysis with $b$-tagged jets sets the strongest limit on the gluino mass. The $\mathrm{SS}+b$ analysis, however, sets limits that are almost independent of mass splitting.
Figure 4 (bottom right) compares the exclusion contours of the T5gg and T5wg models. The limits on the T5gg and T5wg models demonstrate the insensitivity of these photon analyses to the NLSP mass. Also, the requirement on the number of photons (one or two) has little effect on the limit on the T5gg model. The limit on the T5wg model, which has only one signal photon per event, excludes a smaller region than the limit on the T5gg model.

Figure 5 shows values of $[\sigma \times \mathcal{B}]_{\mathrm{UL}}$ for the T1 (T1bbbb), T2 (T2bb), T1tttt, T2tt, TChiSlepSlep, and TChiwz models as functions of the produced particle masses at fixed values of the LSP mass. In the top and middle figures, the LSP mass is fixed at $50 \mathrm{GeV}$, while in the lower figures the LSP is fixed to be massless. Figure 5 also illustrates the method for translating an upper limit on $[\sigma \times \mathcal{B}]_{\mathrm{UL}}$ to a lower limit on the mass of a hypothetical particle. For example, Fig. 5 (top left) displays $[\sigma \times \mathcal{B}]_{\mathrm{UL}}$ for the various analyses that are sensitive to the $\mathrm{T} 1$ and $\mathrm{T} 1 \mathrm{bbbb}$ models. These limits can be compared to $\sigma^{\mathrm{NLO}+\mathrm{NLL}}$ for gluino pair production as a function of 

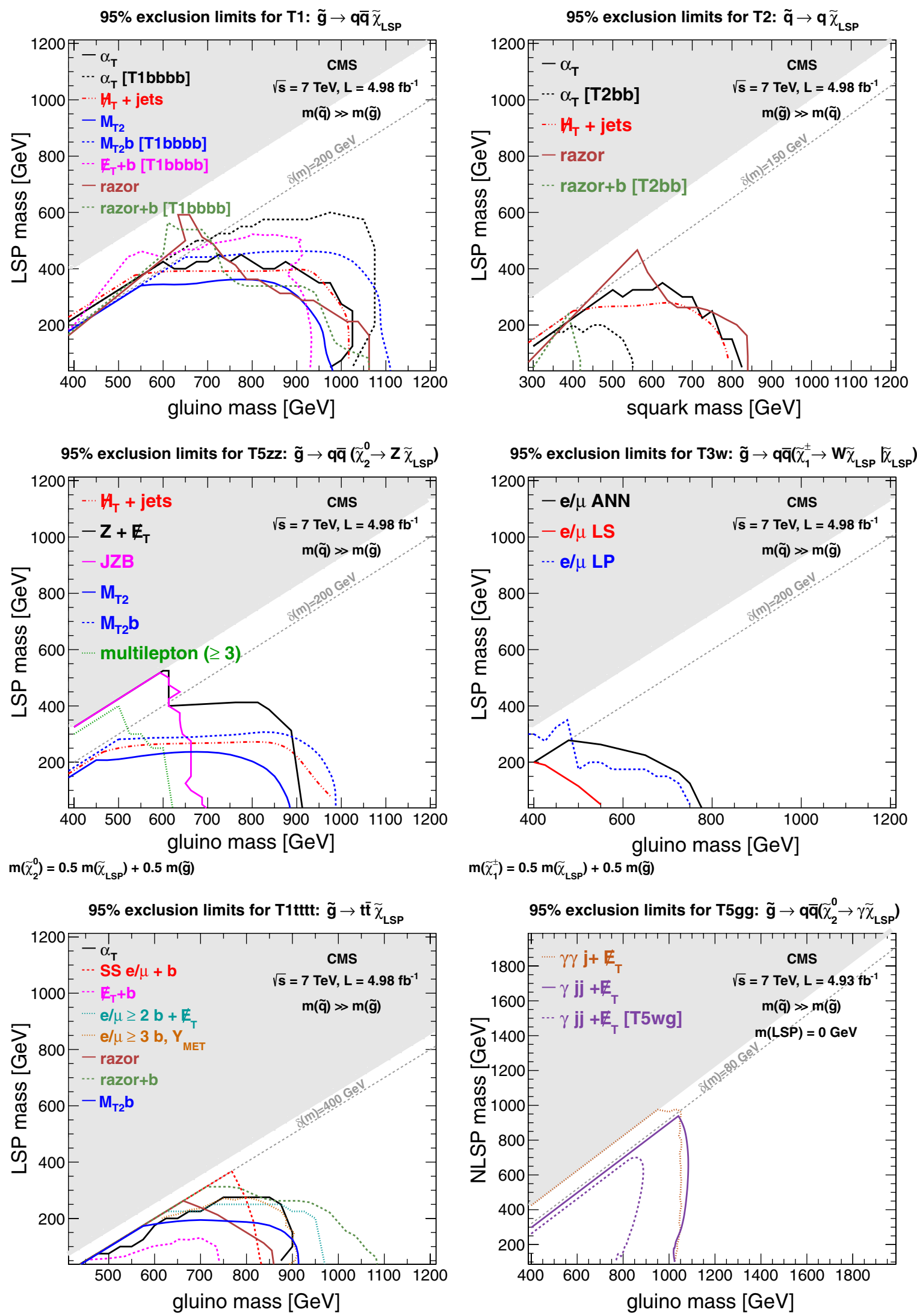

FIG. 4 (color online). The 95\% C.L. exclusion limits on the produced particle and LSP masses in the models T1(T1bbbb), T2(T2bb), $\mathrm{T} 5 \mathrm{zz}, \mathrm{T} 3 \mathrm{w}, \mathrm{T} 1 \mathrm{tttt}$, and $\mathrm{T} 5 \mathrm{gg}(\mathrm{T} 5 \mathrm{wg})$. The grey area represents the region where the decay mode is forbidden. 

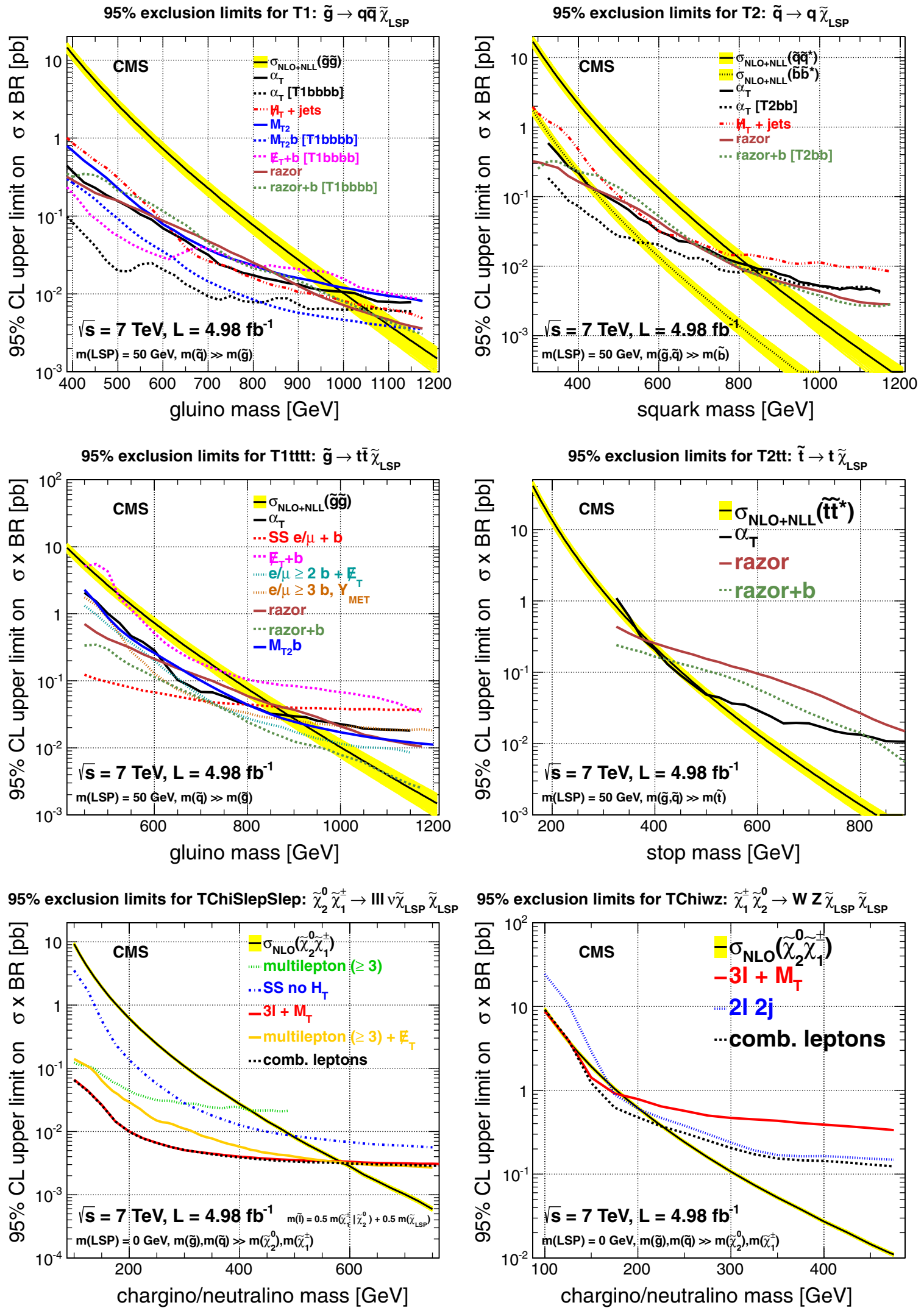

FIG. 5 (color online). The 95\% C.L. exclusion limits and the predicted cross section for the produced particle masses with a fixed LSP mass in the models T1(T1bbbb), T2(T2bb), T1tttt, T2tt, TChiSlepSlep. and TChiwz. 
gluino mass. The intersection of $\sigma^{\mathrm{NLO}+\mathrm{NLL}}$ with $[\sigma \times \mathcal{B}]_{\mathrm{UL}}$ determines a lower limit on the gluino mass. The analyses set a lower limit of approximately $1 \mathrm{TeV}$ on the gluino mass for a LSP mass of $50 \mathrm{GeV}$, corresponding to an upper limit on the cross section of approximately $10 \mathrm{fb}$. This limit assumes $\mathcal{B}=1$ for the decay of each gluino to a lightflavor quark-antiquark pair and the LSP. The (yellow) band on the $\sigma^{\mathrm{NLO}+\mathrm{NLL}}$ curve represents an estimate of the theoretical uncertainties on the cross section calculation. This figure also demonstrates the decrease in $[\sigma \times \mathcal{B}]_{\mathrm{UL}}$ and the increase on the upper limit on the gluino mass for those analyses sensitive to the T1bbbb model.

Similar comparisons can be performed for the different simplified models. For example, Fig. 5 (top right) displays $[\sigma \times \mathcal{B}]_{\mathrm{UL}}$ for the various analyses that are sensitive to the T2 and T2bb models. The analyses set a lower limit of approximately $800 \mathrm{GeV}$ on the squark mass for a LSP mass of $50 \mathrm{GeV}$, corresponding to an upper limit on the cross section of approximately $10 \mathrm{fb}$. This limit assumes there are four squarks with the same mass and that $\mathcal{B}=1$ for the decay of each squark to a light-flavor quark and the LSP. If only bottom squark-antisquark production is considered, and each bottom squark decays to a bottom quark and the LSP, a lower limit of approximately $550 \mathrm{GeV}$ is set on the bottom squark mass for a LSP mass of $50 \mathrm{GeV}$, corresponding to an upper limit on the cross section of approximately $20 \mathrm{fb}$. Figure 5 (bottom left) displays the limits on the model TChiSlepSlep. A chargino mass of approximately $550 \mathrm{GeV}$ is excluded, corresponding to an upper limit on the cross section of approximately $2 \mathrm{fb}$. This limit assumes that $\mathcal{B}=1$ for the decay of the chargino and neutralino to sleptons that further decay to leptons and LSPs. For the model TChiwz, the limit decreases to $220 \mathrm{GeV}$, corresponding to an upper limit on the cross section of approximately $30 \mathrm{fb}$. This limit assumes that $\mathcal{B}=1$ for the decay of the chargino to a $W$ boson and the LSP and the decay of the neutralino to a $Z$ boson and the LSP.

Many of the interpretations presented in Fig. 4 exclude a gluino mass of less than approximately $1 \mathrm{TeV}$ for a range of LSP masses ranging from 200 to $400 \mathrm{GeV}$. However, the exclusion of a particle mass in a simplified model using SUSY cross sections involves assumptions. For example, the $\sigma^{\mathrm{NLO}+\mathrm{NLL}}$ calculation for gluino pair production depends upon the choice of squark masses. If the light-flavor squarks in a specific model, rather than being decoupled, have masses of a few $\mathrm{TeV}$, the predicted gluino cross sections drop significantly due to destructive interference between different amplitudes. The limits on models with cascade decays, T3w, T5lnu, and T5zz, assume a branching fraction of unity for a gluino decay to a chargino or neutralino. However, a realistic MSSM model would contain a degenerate chargino-neutralino pair, reducing the branching fraction to $\frac{1}{2}$ or $\frac{1}{4}$. Furthermore it should be noted that the lower limits on the sparticle masses have been derived for cross sections based on the spin assumed in the CMSSM. Also, the model T2 assumes degenerate copies of left- and right-handed light-flavor squarks, while a realistic model may have a significant mass hierarchy between different squark flavors or eigenstates. As mentioned earlier, the model $\mathrm{T} 2 \mathrm{tt}$ has no spin correlation between the neutralino and the top quark decay products, while such a correlation will arise in the MSSM depending on the mixture of interaction quantum states in the mass quantum states of the top squark and the neutralino. The information contained in this paper and in the supplementary references can be used to set limits if any of these assumptions, or others, are removed or weakened. It must also be noted that the exclusion limits discussed here only serve to broadly summarize simplified model results; the full information on the exclusion power of an analysis in the context of simplified models is contained in the exclusion limits on the production cross section, as shown in Fig. 1. This information is contained in the analysis references. A final caveat can be made regarding the setting of limits in simplified models. Since only one signal process is considered, potential backgrounds are ignored from other signal processes that may arise in a complete model.

\section{SUMMARY}

The simplified model framework is a recently developed method for interpreting the results of searches for new physics. This paper contains a compilation of simplified model interpretations of CMS supersymmetry analyses based on 2011 data. For each simplified model and analysis, an upper limit on the product of the cross section and branching fraction is derived as a function of hypothetical particle masses. Additionally, lower limits on particle masses are determined by comparing the $95 \%$ C.L. upper limit on the product of the cross section and branching fraction to the predicted cross section in supersymmetry for the pair of primary particles. These lower limits depend upon theoretical assumptions that are described earlier in this paper. They should not be regarded as general exclusions on supersymmetric particle masses.

The most stringent results for a few simplified models are summarized here. If the primary particles are gluinos that each decay to quark-antiquark pair and a neutralino, a gluino of mass of approximately $1 \mathrm{TeV}$ is excluded for a neutralino of mass $50 \mathrm{GeV}$. These masses correspond to an upper limit on the gluino pair production cross section of approximately $10 \mathrm{fb}$. The excluded mass increases if each gluino decays to a bottom quark-antiquark pair and a neutralino, while the excluded mass decreases if each gluino decays to a top quark-antiquark pair and a neutralino. The excluded mass also decreases if the gluino undergoes a cascade of decays. If the primary particles are four squark-antisquark pairs, and each squark decays to a 
light-flavor quark and a neutralino, a squark mass of approximately $800 \mathrm{GeV}$ is excluded for a neutralino of mass $50 \mathrm{GeV}$, corresponding to an upper limit on the squarkantisquark production cross section of approximately $10 \mathrm{fb}$. The excluded mass for a single bottom-antibottom squark pair is $550 \mathrm{GeV}$. The comparable exclusion in mass for a single top-antitop squark pair is approximately $150 \mathrm{GeV}$ lower. In the case of the electroweak production of a chargino-neutralino pair, the upper limit on the cross section is approximately 1 order of magnitude higher than the corresponding limit for gluino pair production at the same mass.

The predictions for experimental acceptance and exclusion limits on cross sections presented here for a range of simplified models and mass parameters can be used to constrain other theoretical models and compare different analyses.

\section{ACKNOWLEDGMENTS}

We congratulate our colleagues in the CERN accelerator departments for the excellent performance of the LHC and thank the technical and administrative staffs at CERN and at other CMS institutes for their contributions to the success of the CMS effort. In addition, we gratefully acknowledge the computing centers and personnel of the Worldwide LHC Computing Grid for delivering so effectively the computing infrastructure essential to our analyses. Finally, we acknowledge the enduring support for the construction and operation of the LHC and the CMS detector provided by the following funding agencies: the Austrian Federal Ministry of Science and Research; the Belgian Fonds de la Recherche Scientifique, and Fonds voor Wetenschappelijk Onderzoek; the Brazilian Funding Agencies (CNPq, CAPES, FAPERJ, and FAPESP); the Bulgarian Ministry of Education, Youth and Science; CERN; the Chinese Academy of Sciences, Ministry of Science and Technology, and National Natural Science Foundation of China; the Colombian Funding Agency (COLCIENCIAS); the Croatian Ministry of Science, Education and Sport; the Research Promotion Foundation, Cyprus; the Ministry of Education and Research, Recurrent Financing Contract No. SF0690030s09 and European Regional Development Fund, Estonia; the Academy of Finland, Finnish Ministry of Education and Culture, and Helsinki Institute of Physics; the Institut National de Physique Nucléaire et de Physique des Particules/CNRS, and Commissariat à l'Énergie Atomique et aux Énergies Alternatives/CEA, France; the Bundesministerium für Bildung und Forschung, Deutsche Forschungsgemeinschaft, and
Helmholtz-Gemeinschaft Deutscher Forschungszentren, Germany; the General Secretariat for Research and Technology, Greece; the National Scientific Research Foundation, and National Office for Research and Technology, Hungary; the Department of Atomic Energy and the Department of Science and Technology, India; the Institute for Studies in Theoretical Physics and Mathematics, Iran; the Science Foundation, Ireland; the Istituto Nazionale di Fisica Nucleare, Italy; the Korean Ministry of Education, Science and Technology and the World Class University program of NRF, Republic of Korea; the Lithuanian Academy of Sciences; the Mexican Funding Agencies (CINVESTAV, CONACYT, SEP, and UASLP-FAI); the Ministry of Science and Innovation, New Zealand; the Pakistan Atomic Energy Commission; the Ministry of Science and Higher Education and the National Science Centre, Poland; the Fundação para a Ciência e a Tecnologia, Portugal; JINR (Armenia, Belarus, Georgia, Ukraine, Uzbekistan); the Ministry of Education and Science of the Russian Federation, the Federal Agency of Atomic Energy of the Russian Federation, Russian Academy of Sciences, and the Russian Foundation for Basic Research; the Ministry of Science and Technological Development of Serbia; the Secretaría de Estado de Investigación, Desarrollo e Innovación and Programa Consolider-Ingenio 2010, Spain; the Swiss Funding Agencies (ETH Board, ETH Zurich, PSI, SNF, UniZH, Canton Zurich, and SER); the National Science Council, Taipei; the Thailand Center of Excellence in Physics, the Institute for the Promotion of Teaching Science and Technology of Thailand and the National Science and Technology Development Agency of Thailand; the Scientific and Technical Research Council of Turkey, and Turkish Atomic Energy Authority; the Science and Technology Facilities Council, UK; the U.S. Department of Energy, and the U.S. National Science Foundation. Individuals have received support from the Marie-Curie program and the European Research Council (European Union); the Leventis Foundation; the A.P. Sloan Foundation; the Alexander von Humboldt Foundation; the Belgian Federal Science Policy Office; the Fonds pour la Formation à la Recherche dans l'Industrie et dans l'Agriculture (FRIA-Belgium); the Agentschap voor Innovatie door Wetenschap en Technologie (IWT-Belgium); the Ministry of Education, Youth and Sports (MEYS) of Czech Republic; the Council of Science and Industrial Research, India; the Compagnia di San Paolo (Torino); and the HOMING PLUS program of Foundation for Polish Science, cofinanced from European Union, Regional Development Fund. 
[1] J. Wess and B. Zumino, Nucl. Phys. B70, 39 (1974).

[2] A. H. Chamseddine, R. Arnowitt, and P. Nath, Phys. Rev. Lett. 49, 970 (1982).

[3] R. Arnowitt and P. Nath, Phys. Rev. Lett. 69, 725 (1992).

[4] G. L. Kane, C. F. Kolda, L. Roszkowski, and J. D. Wells, Phys. Rev. D 49, 6173 (1994).

[5] N. Arkani-Hamed, B. Knuteson, S. Mrenna, P. Schuster, J. Thaler, N. Toro, and L.-T. Wang, arXiv:hep-ph/0703088.

[6] J. Alwall, P. C. Schuster, and N. Toro, Phys. Rev. D 79, 075020 (2009).

[7] J. Alwall, M.-P. Le, M. Lisanti, and J. G. Wacker, Phys. Rev. D 79, 015005 (2009).

[8] D. S. M. Alves, E. Izaguirre, and J. G. Wacker, J. High Energy Phys. 10 (2011) 012.

[9] D. Alves et al. (LHC New Physics Working Group), J. Phys. G 39, 105005 (2012).

[10] M. Papucci, J. T. Ruderman, and A. Weiler, J. High Energy Phys. 09 (2012) 035.

[11] R. Mahbubani, M. Papucci, G. Perez, J. T. Ruderman, and A. Weiler, Phys. Rev. Lett. 110, 151804 (2013).

[12] CMS Collaboration, J. High Energy Phys. 01 (2013) 077.

[13] CMS Collaboration, Phys. Rev. Lett. 109, 171803 (2012).

[14] CMS Collaboration, J. High Energy Phys. 10 (2012) 018.

[15] CMS Collaboration, Phys. Rev. D 86, 072010 (2012).

[16] CMS Collaboration, Phys. Rev. Lett. 111, 081802 (2013).

[17] CMS Collaboration, Eur. Phys. J. C 73, 2404 (2013).

[18] CMS Collaboration, Phys. Rev. D 87, 052006 (2013).

[19] CMS Collaboration, Phys. Rev. Lett. 109, 071803 (2012).

[20] CMS Collaboration, J. High Energy Phys. 08 (2012) 110.

[21] CMS Collaboration, Phys. Lett. B 718, 815 (2013).

[22] CMS Collaboration, Phys. Rev. D 87, 072001 (2013).

[23] CMS Collaboration, J. High Energy Phys. 06 (2012) 169.

[24] CMS Collaboration, J. High Energy Phys. 11 (2012) 147.

[25] CMS Collaboration, Phys. Lett. B 716, 260 (2012).

[26] CMS Collaboration, J. High Energy Phys. 03 (2013) 111.

[27] CMS Collaboration, J. High Energy Phys. 08 (2011) 155.

[28] ATLAS Collaboration, Phys. Rev. D 87, 012008 (2013).
[29] ATLAS Collaboration, Eur. Phys. J. C 72, 2174 (2012).

[30] ATLAS Collaboration, Phys. Lett. B 718, 879 (2013).

[31] ATLAS Collaboration, Phys. Lett. B 718, 841 (2013).

[32] ATLAS Collaboration, Phys. Rev. D 85, 112006 (2012).

[33] ATLAS Collaboration, J. High Energy Phys. 07 (2012) 167.

[34] ATLAS Collaboration, Phys. Rev. D 86, 092002 (2012).

[35] CMS Collaboration, JINST 3, S08004 (2008).

[36] CMS Collaboration, CMS Physics Analysis Summary No. CMS-PAS-SMP-12-008, 2012.

[37] L. Randall and D. Tucker-Smith, Phys. Rev. Lett. 101, 221803 (2008).

[38] A. Barr, C. Lester, and P. Stephens, J. Phys. G 29, 2343 (2003).

[39] C. G. Lester and D. J. Summers, Phys. Lett. B 463, 99 (1999).

[40] C. Rogan, arXiv:1006.2727.

[41] P. Meade, N. Seiberg, and D. Shih, Prog. Theor. Phys. Suppl. 177, 143 (2009).

[42] T. Sjöstrand, S. Mrenna, and P.Z. Skands, J. High Energy Phys. 05 (2006) 026.

[43] W. Beenakker, R. Höpker, M. Spira, and P. M. Zerwas, Nucl. Phys. B492, 51 (1997).

[44] A. Kulesza and L. Motyka, Phys. Rev. Lett. 102, 111802 (2009).

[45] A. Kulesza and L. Motyka, Phys. Rev. D 80, 095004 (2009).

[46] W. Beenakker, S. Brensing, M. Krämer, A. Kulesza, E. Laenen, and I. Niessen, J. High Energy Phys. 12 (2009) 041.

[47] W. Beenakker, S. Brensing, M. Krämer, A. Kulesza, E. Laenen, L. Motyka, and I. Niessen, Int. J. Mod. Phys. A 26, 2637 (2011).

[48] M. Krämer, A. Kulesza, R. van der Leeuw, M. Mangano, S. Padhi, T. Plehn, and P. Xavier, arXiv:1206.2892.

[49] A. L. Read, J. Phys. G 28, 2693 (2002).

[50] T. Junk, Nucl. Instrum. Methods Phys. Res., Sect. A 434, 435 (1999).

S. Chatrchyan, ${ }^{1}$ V. Khachatryan, ${ }^{1}$ A. M. Sirunyan, ${ }^{1}$ A. Tumasyan, ${ }^{1}$ W. Adam,${ }^{2}$ E. Aguilo, ${ }^{2}$ T. Bergauer, ${ }^{2}$ M. Dragicevic, ${ }^{2}$ J. Erö, ${ }^{2}$ C. Fabjan, ${ }^{2, b}$ M. Friedl, ${ }^{2}$ R. Frühwirth, ${ }^{2, b}$ V. M. Ghete, ${ }^{2}$ N. Hörmann, ${ }^{2}$ J. Hrubec, ${ }^{2}$ M. Jeitler, ${ }^{2, \mathrm{~b}}$ W. Kiesenhofer, ${ }^{2}$ V. Knünz, ${ }^{2}$ M. Krammer, ${ }^{2, \mathrm{~b}}$ I. Krätschmer, ${ }^{2}$ D. Liko, ${ }^{2}$ I. Mikulec, ${ }^{2}$ M. Pernicka, ${ }^{2, \mathrm{a}}$ D. Rabady, ${ }^{2, \mathrm{c}}$ B. Rahbaran, ${ }^{2}$ C. Rohringer, ${ }^{2}$ H. Rohringer, ${ }^{2}$ R. Schöfbeck, ${ }^{2}$ J. Strauss, ${ }^{2}$ A. Taurok,${ }^{2}$ W. Waltenberger, ${ }^{2}$ C.-E. Wulz, ${ }^{2, b}$ V. Mossolov, ${ }^{3}$ N. Shumeiko, ${ }^{3}$ J. Suarez Gonzalez, ${ }^{3}$ M. Bansal, ${ }^{4}$ S. Bansal, ${ }^{4}$ T. Cornelis, ${ }^{4}$ E. A. De Wolf, ${ }^{4}$ X. Janssen, ${ }^{4}$ S. Luyckx,${ }^{4}$ L. Mucibello, ${ }^{4}$ S. Ochesanu, ${ }^{4}$ B. Roland, ${ }^{4}$ R. Rougny, ${ }^{4}$ M. Selvaggi, ${ }^{4}$ H. Van Haevermaet, ${ }^{4}$ P. Van Mechelen, ${ }^{4}$ N. Van Remortel, ${ }^{4}$ A. Van Spilbeeck, ${ }^{4}$ F. Blekman, ${ }^{5}$ S. Blyweert, ${ }^{5}$ J. D'Hondt, ${ }^{5}$ R. Gonzalez Suarez, ${ }^{5}$ A. Kalogeropoulos, ${ }^{5}$ M. Maes, ${ }^{5}$ A. Olbrechts, ${ }^{5}$ W. Van Doninck, ${ }^{5}$ P. Van Mulders, ${ }^{5}$ G. P. Van Onsem, ${ }^{5}$ I. Villella, ${ }^{5}$ B. Clerbaux, ${ }^{6}$ G. De Lentdecker, ${ }^{6}$ V. Dero, ${ }_{6}^{6}$ A. P. R. Gay, ${ }^{6}$ T. Hreus, ${ }^{6}$ A. Léonard, ${ }^{6}$ P.E. Marage, ${ }^{6}$ A. Mohammadi, ${ }^{6}$ T. Reis,${ }^{6}$ L. Thomas,${ }^{6}$ C. Vander Velde,${ }^{6}$ P. Vanlaer, ${ }^{6}$ J. Wang, ${ }^{6}$ V. Adler, ${ }^{7}$ K. Beernaert, ${ }^{7}$ A. Cimmino, ${ }^{7}$ S. Costantini, ${ }^{7}$ G. Garcia, ${ }^{7}$ M. Grunewald, ${ }^{7}$ B. Klein, ${ }^{7}$ J. Lellouch, ${ }^{7}$ A. Marinov, ${ }^{7}$ J. Mccartin, ${ }^{7}$ A. A. Ocampo Rios, ${ }^{7}$ D. Ryckbosch, ${ }^{7}$ N. Strobbe,${ }^{7}$ F. Thyssen, ${ }^{7}$ M. Tytgat, ${ }^{7}$ S. Walsh, ${ }^{7}$ E. Yazgan, ${ }^{7}$ N. Zaganidis, ${ }^{7}$ S. Basegmez, ${ }^{8}$ G. Bruno,${ }^{8}$ R. Castello, ${ }^{8}$ L. Ceard,${ }^{8}$ C. Delaere, ${ }^{8}$ T. du Pree,${ }^{8}$ D. Favart, ${ }^{8}$ L. Forthomme, ${ }^{8}$ A. Giammanco, ${ }^{8, \mathrm{~d}}$ J. Hollar, ${ }^{8}$ V. Lemaitre, ${ }^{8}$ J. Liao, ${ }^{8}$ O. Militaru, ${ }^{8}$ C. Nuttens, ${ }^{8}$ D. Pagano,${ }^{8}$ A. Pin, ${ }^{8}$

K. Piotrzkowski, ${ }^{8}$ J. M. Vizan Garcia,${ }^{8}$ N. Beliy, ${ }^{9}$ T. Caebergs, ${ }^{9}$ E. Daubie, ${ }^{9}$ G. H. Hammad, ${ }^{9}$ G. A. Alves, ${ }^{10}$ M. Correa Martins Junior, ${ }^{10}$ T. Martins, ${ }^{10}$ M. E. Pol, ${ }^{10}$ M. H. G. Souza, ${ }^{10}$ W. L. Aldá Júnior, ${ }^{11}$ W. Carvalho, ${ }^{11}$ A. Custódio, ${ }^{11}$ E. M. Da Costa, ${ }^{11}$ D. De Jesus Damiao, ${ }^{11}$ C. De Oliveira Martins, ${ }^{11}$ S. Fonseca De Souza, ${ }^{11}$ H. Malbouisson, ${ }^{11}$ M. Malek, ${ }^{11}$ D. Matos Figueiredo, ${ }^{11}$ L. Mundim, ${ }^{11}$ H. Nogima, ${ }^{11}$ W. L. Prado Da Silva, ${ }^{11}$ 
A. Santoro, ${ }^{11}$ L. Soares Jorge, ${ }^{11}$ A. Sznajder, ${ }^{11}$ A. Vilela Pereira, ${ }^{11}$ T. S. Anjos, ${ }^{12 b}$ C. A. Bernardes, ${ }^{12 b}$ F. A. Dias, ${ }^{12 a, e}$ T. R. Fernandez Perez Tomei, ${ }^{12 a}$ E. M. Gregores, ${ }^{12 b}$ C. Lagana, ${ }^{12 a}$ F. Marinho, ${ }^{12 a}$ P. G. Mercadante, ${ }^{12 b}$ S. F. Novaes, ${ }^{12 a}$ Sandra S. Padula, ${ }^{12 a}$ V. Genchev,${ }^{13, c}$ P. Iaydjiev, ${ }^{13, c}$ S. Piperov, ${ }^{13}$ M. Rodozov,${ }^{13}$ S. Stoykova, ${ }^{13}$ G. Sultanov, ${ }^{13}$ V. Tcholakov,${ }^{13}$ R. Trayanov,${ }^{13}$ M. Vutova, ${ }^{13}$ A. Dimitrov,${ }^{14}$ R. Hadjiiska, ${ }^{14}$ V. Kozhuharov, ${ }^{14}$ L. Litov, ${ }^{14}$ B. Pavlov, ${ }^{14}$ P. Petkov, ${ }^{14}$ J. G. Bian, ${ }^{15}$ G. M. Chen, ${ }^{15}$ H. S. Chen, ${ }^{15}$ C. H. Jiang, ${ }^{15}$ D. Liang, ${ }^{15}$ S. Liang, ${ }^{15}$ X. Meng, ${ }^{15}$ J. Tao, ${ }^{15}$ J. Wang, ${ }^{15}$ X. Wang, ${ }^{15}$ Z. Wang, ${ }^{15}$ H. Xiao, ${ }^{15}$ M. Xu,${ }^{15}$ J. Zang, ${ }^{15}$ Z. Zhang, ${ }^{15}$ C. Asawatangtrakuldee, ${ }^{16}$ Y. Ban ${ }^{16}$ Y. Guo, ${ }^{16}$ W. Li, ${ }^{16}$ S. Liu, ${ }^{16}$ Y. Mao, ${ }^{16}$ S. J. Qian, ${ }^{16}$ H. Teng, ${ }^{16}$ D. Wang, ${ }^{16}$ L. Zhang, ${ }^{16}$ W. Zou, ${ }^{16}$ C. Avila, ${ }^{17}$ J. P. Gomez, ${ }^{17}$ B. Gomez Moreno, ${ }^{17}$ A. F. Osorio Oliveros, ${ }^{17}$ J. C. Sanabria, ${ }^{17}$ N. Godinovic, ${ }^{18}$ D. Lelas, ${ }^{18}$ R. Plestina,${ }^{18, \mathrm{f}}$ D. Polic,${ }^{18}$ I. Puljak, ${ }^{18, \mathrm{c}}$ Z. Antunovic, ${ }^{19}$ M. Kovac, ${ }^{19}$ V. Brigljevic,${ }^{20}$ S. Duric,${ }^{20}$ K. Kadija, ${ }^{20}$ J. Luetic, ${ }^{20}$ D. Mekterovic,${ }^{20}$ S. Morovic,${ }^{20}$ A. Attikis, ${ }^{21}$ M. Galanti, ${ }^{21}$ G. Mavromanolakis, ${ }^{21}$ J. Mousa, ${ }^{21}$ C. Nicolaou, ${ }^{21}$ F. Ptochos,${ }^{21}$ P. A. Razis, ${ }^{21}$ M. Finger, ${ }^{22}$ M. Finger, Jr. ${ }^{22}$ Y. Assran, ${ }^{23, g}$ S. Elgammal, ${ }^{23, h}$ A. Ellithi Kamel, ${ }^{23, \mathrm{i}}$ M. A. Mahmoud,${ }^{23, \mathrm{j}}$ A. Mahrous,${ }^{23, \mathrm{k}}$ A. Radi ${ }^{23,1, \mathrm{~m}}$ M. Kadastik, ${ }^{24}$ M. Müntel, ${ }^{24}$ M. Raidal, ${ }^{24}$ L. Rebane, ${ }^{24}$ A. Tiko, ${ }^{24}$ P. Eerola, ${ }^{25}$ G. Fedi, ${ }^{25}$ M. Voutilainen,${ }^{25}$ J. Härkönen ${ }^{26}$ A. Heikkinen, ${ }^{26}$ V. Karimäki ${ }^{26}$ R. Kinnunen, ${ }^{26}$ M. J. Kortelainen, ${ }^{26}$ T. Lampén, ${ }^{26}$ K. Lassila-Perini, ${ }^{26}$ S. Lehti, ${ }^{26}$ T. Lindén, ${ }^{26}$ P. Luukka ${ }^{26}$ T. Mäenpää, ${ }^{26}$ T. Peltola, ${ }^{26}$ E. Tuominen, ${ }^{26}$ J. Tuominiemi, ${ }^{26}$ E. Tuovinen, ${ }^{26}$ D. Ungaro, ${ }^{26}$ L. Wendland, ${ }^{26}$ K. Banzuzi, ${ }^{27}$ A. Karjalainen, ${ }^{27}$ A. Korpela, ${ }^{27}$ T. Tuuva, ${ }^{27}$ M. Besancon, ${ }^{28}$ S. Choudhury, ${ }^{28}$ M. Dejardin, ${ }^{28}$ D. Denegri, ${ }^{28}$ B. Fabbro, ${ }^{28}$ J. L. Faure,${ }^{28}$ F. Ferri, ${ }^{28}$ S. Ganjour, ${ }^{28}$ A. Givernaud, ${ }^{28}$ P. Gras,${ }^{28}$

G. Hamel de Monchenault, ${ }^{28}$ P. Jarry, ${ }^{28}$ E. Locci, ${ }^{28}$ J. Malcles, ${ }^{28}$ L. Millischer, ${ }^{28}$ A. Nayak, ${ }^{28}$ J. Rander, ${ }^{28}$ A. Rosowsky, ${ }^{28}$ M. Titov, ${ }^{28}$ S. Baffioni, ${ }^{29}$ F. Beaudette, ${ }^{29}$ L. Benhabib,${ }^{29}$ L. Bianchini, ${ }^{29}$ M. Bluj, ${ }^{29, n}$ P. Busson, ${ }^{29}$ C. Charlot,${ }^{29}$ N. Daci, ${ }^{29}$ T. Dahms,${ }^{29}$ M. Dalchenko, ${ }^{29}$ L. Dobrzynski, ${ }^{29}$ A. Florent, ${ }^{29}$ R. Granier de Cassagnac, ${ }^{29}$ M. Haguenauer, ${ }^{29}$ P. Miné, ${ }^{29}$ C. Mironov, ${ }^{29}$ I. N. Naranjo, ${ }^{29}$ M. Nguyen, ${ }^{29}$ C. Ochando, ${ }^{29}$ P. Paganini, ${ }^{29}$ D. Sabes, ${ }^{29}$ R. Salerno, ${ }^{29}$ Y. Sirois, ${ }^{29}$ C. Veelken, ${ }^{29}$ A. Zabi, ${ }^{29}$ J.-L. Agram,,${ }^{30, o}$ J. Andrea, ${ }^{30}$ D. Bloch,${ }^{30}$ D. Bodin, ${ }^{30}$ J.-M. Brom, ${ }^{30}$ M. Cardaci, ${ }^{30}$ E. C. Chabert,${ }^{30}$ C. Collard ${ }^{30}$ E. Conte,${ }^{30, o}$ F. Drouhin, ${ }^{30, o}$ J.-C. Fontaine, ${ }^{30, o}$ D. Gelé, ${ }^{30}$ U. Goerlach, ${ }^{30}$ P. Juillot, ${ }^{30}$ A.-C. Le Bihan, ${ }^{30}$ P. Van Hove, ${ }^{30}$ F. Fassi, ${ }^{31}$ D. Mercier, ${ }^{31}$ S. Beauceron, ${ }^{32}$ N. Beaupere, ${ }^{32}$ O. Bondu, ${ }^{32}$ G. Boudoul, ${ }^{32}$ S. Brochet, ${ }^{32}$ J. Chasserat, ${ }^{32}$ R. Chierici, ${ }^{32, c}$ D. Contardo, ${ }^{32}$ P. Depasse, ${ }^{32}$ H. El Mamouni, ${ }^{32}$ J. Fay, ${ }^{32}$ S. Gascon, ${ }^{32}$ M. Gouzevitch, ${ }^{32}$ B. Ille, ${ }^{32}$ T. Kurca, ${ }^{32}$ M. Lethuillier, ${ }^{32}$ L. Mirabito, ${ }^{32}$ S. Perries, ${ }^{32}$ L. Sgandurra, ${ }^{32}$ V. Sordini, ${ }^{32}$ Y. Tschudi, ${ }^{32}$ P. Verdier, ${ }^{32}$ S. Viret, ${ }^{32}$ Z. Tsamalaidze, ${ }^{33, p}$ C. Autermann,${ }^{34}$ S. Beranek, ${ }^{34}$ B. Calpas, ${ }^{34}$ M. Edelhoff, ${ }^{34}$ L. Feld,${ }^{34}$ N. Heracleous, ${ }^{34}$ O. Hindrichs,${ }^{34}$ R. Jussen, ${ }^{34}$ K. Klein, ${ }^{34}$ J. Merz, ${ }^{34}$ A. Ostapchuk, ${ }^{34}$ A. Perieanu, ${ }^{34}$ F. Raupach, ${ }^{34}$ J. Sammet, ${ }^{34}$ S. Schael, ${ }^{34}$ D. Sprenger, ${ }^{34}$ H. Weber, ${ }^{34}$ B. Wittmer, ${ }^{34}$ V. Zhukov, ${ }^{34, q}$ M. Ata, ${ }^{35}$ J. Caudron, ${ }^{35}$ E. Dietz-Laursonn, ${ }^{35}$ D. Duchardt, ${ }^{35}$ M. Erdmann, ${ }^{35}$

R. Fischer, ${ }^{35}$ A. Güth, ${ }^{35}$ T. Hebbeker, ${ }^{35}$ C. Heidemann, ${ }^{35}$ K. Hoepfner, ${ }^{35}$ D. Klingebiel, ${ }^{35}$ P. Kreuzer,${ }^{35}$ M. Merschmeyer, ${ }^{35}$ A. Meyer, ${ }^{35}$ M. Olschewski, ${ }^{35}$ P. Papacz, ${ }^{35}$ H. Pieta, ${ }^{35}$ H. Reithler, ${ }^{35}$ S. A. Schmitz, ${ }^{35}$ L. Sonnenschein, ${ }^{35}$ J. Steggemann, ${ }^{35}$ D. Teyssier, ${ }^{35}$ S. Thüer, ${ }^{35}$ M. Weber,${ }^{35}$ M. Bontenackels, ${ }^{36}$ V. Cherepanov, ${ }^{36}$ Y. Erdogan, ${ }^{36}$ G. Flügge, ${ }^{36}$ H. Geenen,${ }^{36}$ M. Geisler, ${ }^{36}$ W. Haj Ahmad,${ }^{36}$ F. Hoehle, ${ }^{36}$ B. Kargoll, ${ }^{36}$ T. Kress, ${ }^{36}$ Y. Kuessel, ${ }^{36}$ J. Lingemann, ${ }^{36, c}$ A. Nowack, ${ }^{36}$ L. Perchalla, ${ }^{36}$ O. Pooth, ${ }^{36}$ P. Sauerland, ${ }^{36}$ A. Stahl, ${ }^{36}$ M. Aldaya Martin, ${ }^{37}$ J. Behr,,${ }^{37}$ W. Behrenhoff,,${ }^{37}$ U. Behrens, ${ }^{37}$ M. Bergholz, ${ }^{37, \mathrm{r}}$ A. Bethani, ${ }^{37}$ K. Borras,${ }^{37}$ A. Burgmeier, ${ }^{37}$ A. Cakir,${ }^{37}$ L. Calligaris,${ }^{37}$ A. Campbell, ${ }^{37}$ E. Castro, ${ }^{37}$ F. Costanza,,${ }^{37}$ D. Dammann, ${ }^{37}$ C. Diez Pardos, ${ }^{37}$ G. Eckerlin, ${ }^{37}$ D. Eckstein, ${ }^{37}$ G. Flucke, ${ }^{37}$ A. Geiser, ${ }^{37}$ I. Glushkov, ${ }^{37}$ P. Gunnellini, ${ }^{37}$ S. Habib,${ }^{37}$ J. Hauk, ${ }^{37}$ G. Hellwig, ${ }^{37}$ H. Jung, ${ }^{37}$ M. Kasemann, ${ }^{37}$ P. Katsas, ${ }^{37}$ C. Kleinwort, ${ }^{37}$ H. Kluge, ${ }^{37}$ A. Knutsson, ${ }^{37}$ M. Krämer, ${ }^{37}$ D. Krücker, ${ }^{37}$ E. Kuznetsova, ${ }^{37}$ W. Lange, ${ }^{37}$ J. Leonard, ${ }^{37}$ W. Lohmann, ${ }^{37, r}$ B. Lutz, ${ }^{37}$ R. Mankel, ${ }^{37}$ I. Marfin, ${ }^{37}$ M. Marienfeld,${ }^{37}$ I.-A. Melzer-Pellmann, ${ }^{37}$ A. B. Meyer, ${ }^{37}$ J. Mnich, ${ }^{37}$ A. Mussgiller, ${ }^{37}$

S. Naumann-Emme, ${ }^{37}$ O. Novgorodova, ${ }^{37}$ J. Olzem, ${ }^{37}$ H. Perrey,${ }^{37}$ A. Petrukhin, ${ }^{37}$ D. Pitzl, ${ }^{37}$ A. Raspereza,${ }^{37}$ P. M. Ribeiro Cipriano, ${ }^{37}$ C. Riedl,${ }^{37}$ E. Ron, ${ }^{37}$ M. Rosin, ${ }^{37}$ J. Salfeld-Nebgen,${ }^{37}$ R. Schmidt, ${ }^{37, r}$

T. Schoerner-Sadenius, ${ }^{37}$ N. Sen, ${ }^{37}$ A. Spiridonov, ${ }^{37}$ M. Stein, ${ }^{37}$ R. Walsh, ${ }^{37}$ C. Wissing, ${ }^{37}$ V. Blobel,${ }^{38}$ H. Enderle, ${ }^{38}$ J. Erfle, ${ }^{38}$ U. Gebbert, ${ }^{38}$ M. Görner, ${ }^{38}$ M. Gosselink, ${ }^{38}$ J. Haller, ${ }^{38}$ T. Hermanns, ${ }^{38}$ R. S. Höing, ${ }^{38}$ K. Kaschube, ${ }^{38}$ G. Kaussen, ${ }^{38}$ H. Kirschenmann, ${ }^{38}$ R. Klanner, ${ }^{38}$ J. Lange, ${ }^{38}$ F. Nowak, ${ }^{38}$ T. Peiffer, ${ }^{38}$ N. Pietsch, ${ }^{38}$ D. Rathjens, ${ }^{38}$ C. Sander, ${ }^{38}$ H. Schettler, ${ }^{38}$ P. Schleper, ${ }^{38}$ E. Schlieckau, ${ }^{38}$ A. Schmidt, ${ }^{38}$ M. Schröder, ${ }^{38}$ T. Schum, ${ }^{38}$ M. Seidel, ${ }^{38}$ J. Sibille, ${ }^{38, s}$ V. Sola,${ }^{38}$ H. Stadie,${ }^{38}$ G. Steinbrück, ${ }^{38}$ J. Thomsen, ${ }^{38}$ L. Vanelderen,${ }^{38}$ C. Barth, ${ }^{39}$ J. Berger,${ }^{39}$ C. Böser, ${ }^{39}$ T. Chwalek, ${ }^{39}$ W. De Boer, ${ }^{39}$ A. Descroix,${ }^{39}$ A. Dierlamm, ${ }^{39}$ M. Feindt, ${ }^{39}$ M. Guthoff,,${ }^{39, c}$ C. Hackstein, ${ }^{39}$

F. Hartmann, ${ }^{39, \mathrm{c}}$ T. Hauth, ${ }^{39, \mathrm{c}}$ M. Heinrich, ${ }^{39}$ H. Held ${ }^{39}$ K. H. Hoffmann, ${ }^{39}$ U. Husemann, ${ }^{39}$ I. Katkov, ${ }^{39, q}$ J. R. Komaragiri, ${ }^{39}$ P. Lobelle Pardo, ${ }^{39}$ D. Martschei, ${ }^{39}$ S. Mueller, ${ }^{39}$ Th. Müller, ${ }^{39}$ M. Niegel, ${ }^{39}$ A. Nürnberg, ${ }^{39}$ 
O. Oberst,${ }^{39}$ A. Oehler, ${ }^{39}$ J. Ott, ${ }^{39}$ G. Quast,${ }^{39}$ K. Rabbertz,${ }^{39}$ F. Ratnikov, ${ }^{39}$ N. Ratnikova, ${ }^{39}$ S. Röcker, ${ }^{39}$

F.-P. Schilling, ${ }^{39}$ G. Schott,${ }^{39}$ H. J. Simonis, ${ }^{39}$ F. M. Stober,${ }^{39}$ D. Troendle, ${ }^{39}$ R. Ulrich, ${ }^{39}$ J. Wagner-Kuhr, ${ }^{39}$

S. Wayand, ${ }^{39}$ T. Weiler, ${ }^{39}$ M. Zeise, ${ }^{39}$ G. Anagnostou, ${ }^{40}$ G. Daskalakis, ${ }^{40}$ T. Geralis, ${ }^{40}$ S. Kesisoglou, ${ }^{40}$ A. Kyriakis, ${ }^{40}$

D. Loukas, ${ }^{40}$ I. Manolakos, ${ }^{40}$ A. Markou, ${ }^{40}$ C. Markou, ${ }^{40}$ E. Ntomari,${ }^{40}$ L. Gouskos,${ }^{41}$ T. J. Mertzimekis,${ }^{41}$

A. Panagiotou, ${ }^{41}$ N. Saoulidou, ${ }^{41}$ I. Evangelou, ${ }^{42}$ C. Foudas,${ }^{42}$ P. Kokkas, ${ }^{42}$ N. Manthos,${ }^{42}$ I. Papadopoulos, ${ }^{42}$ V. Patras, ${ }^{42}$ G. Bencze, ${ }^{43}$ C. Hajdu, ${ }^{43}$ P. Hidas,${ }^{43}$ D. Horvath,${ }^{43, t}$ F. Sikler, ${ }^{43}$ V. Veszpremi, ${ }^{43}$ G. Vesztergombi, ${ }^{43, u}$ N. Beni, ${ }^{44}$ S. Czellar, ${ }^{44}$ J. Molnar, ${ }^{44}$ J. Palinkas, ${ }^{44}$ Z. Szillasi, ${ }^{44}$ J. Karancsii, ${ }^{45}$ P. Raics, ${ }^{45}$ Z. L. Trocsanyi, ${ }^{45}$ B. Ujvari, ${ }^{45}$ S. B. Beri, ${ }^{46}$ V. Bhatnagar, ${ }^{46}$ N. Dhingra, ${ }^{46}$ R. Gupta, ${ }^{46}$ M. Kaur, ${ }^{46}$ M. Z. Mehta, ${ }^{46}$ N. Nishu,${ }^{46}$ L. K. Saini ${ }^{46}$ A. Sharma, ${ }^{46}$ J. B. Singh, ${ }^{46}$ Ashok Kumar, ${ }^{47}$ Arun Kumar, ${ }^{47}$ S. Ahuja ${ }^{47}$ A. Bhardwaj, ${ }^{47}$ B. C. Choudhary, ${ }^{47}$ S. Malhotra, ${ }^{47}$ M. Naimuddin, ${ }^{47}$ K. Ranjan, ${ }^{47}$ V. Sharma,${ }^{47}$ R. K. Shivpuri, ${ }^{47}$ S. Banerjee, ${ }^{48}$ S. Bhattacharyaa ${ }^{48}$ S. Dutta, ${ }^{48}$ B. Gomber, ${ }^{48}$ Sa. Jain ${ }^{48}$ Sh. Jain, ${ }^{48}$ R. Khurana, ${ }^{48}$ S. Sarkar, ${ }^{48}$ M. Sharan, ${ }^{48}$ A. Abdulsalam, ${ }^{49}$ D. Dutta, ${ }^{49}$ S. Kailas, ${ }^{49}$ V. Kumar, ${ }^{49}$ A. K. Mohanty, ${ }^{49, c}$ L. M. Pant, ${ }^{49}$ P. Shukla, ${ }^{49}$ T. Aziz ${ }^{50}$ S. Ganguly, ${ }^{50}$ M. Guchait, ${ }^{50, v}$ A. Gurtu, ${ }^{50, w}$ M. Maity, ${ }^{50, x}$ G. Majumder, ${ }^{50}$ K. Mazumdar, ${ }^{50}$ G. B. Mohanty, ${ }^{50}$ B. Parida ${ }^{50}$ K. Sudhakar, ${ }^{50}$ N. Wickramage, ${ }^{50}$ S. Banerjee, ${ }^{51}$ S. Dugad,${ }^{51}$ H. Arfaei,${ }^{52, y}$ H. Bakhshiansohi, ${ }^{52}$ S. M. Etesami, ${ }^{52, z}$ A. Fahim ${ }^{52, y}$ M. Hashemi,${ }^{52, a a}$ H. Hesari, ${ }^{52}$ A. Jafari, ${ }^{52}$ M. Khakzad, ${ }^{52}$

M. Mohammadi Najafabadi, ${ }^{52}$ S. Paktinat Mehdiabadi, ${ }^{52}$ B. Safarzadeh, ${ }^{52, b b}$ M. Zeinali, ${ }^{52}$ M. Abbrescia,,${ }^{53 a, 53 b}$ L. Barbone, ${ }^{53 a, 53 b}$ C. Calabria, ${ }^{53 a, 53 b, c}$ S. S. Chhibra, ${ }^{53 a, 53 b}$ A. Colaleo, ${ }^{53 a}$ D. Creanza, ${ }^{53 a, 53 c}$ N. De Filippis, ${ }^{53 a, 53 c, c}$ M. De Palma, ${ }^{53 a, 53 b}$ L. Fiore, ${ }^{53 a}$ G. Iaselli, ${ }^{53 a, 53 c}$ G. Maggi, ${ }^{53 a, 53 c}$ M. Maggi, ${ }^{53 a}$ B. Marangelli, ${ }^{53 a, 53 b}$ S. My, ${ }^{53 a, 53 c}$ S. Nuzzo, ${ }^{53 a, 53 b}$ N. Pacifico, ${ }^{53 a}$ A. Pompili, ${ }^{53 a, 53 b}$ G. Pugliese, ${ }^{53 a, 53 c}$ G. Selvaggi, ${ }^{53 a, 53 b}$ L. Silvestris, ${ }^{53 a}$ G. Singh, ${ }^{53 a, 53 b}$ R. Venditti, ${ }^{53 a, 53 b}$ P. Verwilligen, ${ }^{53 a}$ G. Zito, ${ }^{53 a}$ G. Abbiendi, ${ }^{54 a}$ A. C. Benvenuti, ${ }^{54 a}$ D. Bonacorsi, ${ }^{54 a, 54 b}$ S. Braibant-Giacomelli, ${ }^{54 a, 54 b}$ L. Brigliadori, ${ }^{54,54 b}$ P. Capiluppi, ${ }^{54 a, 54 b}$ A. Castro, ${ }^{54 a, 54 b}$ F. R. Cavallo, ${ }^{54 a}$ M. Cuffiani, ${ }^{54 a, 54 b}$ G. M. Dallavalle, ${ }^{54 a}$ F. Fabbri, ${ }^{54 a}$ A. Fanfani, ${ }^{54 a, 54 b}$ D. Fasanella, ${ }^{54 a, 54 b}$ P. Giacomelli, ${ }^{54 a}$ C. Grandi, ${ }^{54 a}$ L. Guiducci, ${ }^{54 a, 54 b}$ S. Marcellini, ${ }^{54 a}$ G. Masetti, ${ }^{54 a}$ M. Meneghelli, ${ }^{54 a, 54 b, c}$ A. Montanari, ${ }^{54 a}$ F. L. Navarria, ${ }^{54 a, 54 b}$ F. Odorici, ${ }^{54 a}$ A. Perrotta, ${ }^{54 a}$ F. Primavera, ${ }^{54 a, 54 b}$ A. M. Rossi, ${ }^{54 a, 54 b}$ T. Rovelli, ${ }^{54 a, 54 b}$ G. P. Siroli, ${ }^{54 a, 54 b}$ N. Tosi, ${ }^{54 a}$ R. Travaglini, ${ }^{54 a, 54 b}$ S. Albergo, ${ }^{55 a, 55 b}$ G. Cappello, ${ }^{55 a, 55 b}$ M. Chiorboli, ${ }^{55 a, 55 b}$ S. Costa, ${ }^{55 a, 55 b}$ R. Potenza, ${ }^{55 a, 55 b}$ A. Tricomi, ${ }^{55 a, 55 b}$ C. Tuve, ${ }^{55 a, 55 b}$ G. Barbagli, ${ }^{56 a}$ V. Ciulli, ${ }^{56 a, 56 b}$ C. Civinini, ${ }^{56 a}$ R. D'Alessandro, ${ }^{56 a, 56 b}$ E. Focardi, ${ }^{56 a, 56 b}$ S. Frosali, ${ }^{56 a, 56 b}$ E. Gallo, ${ }^{56 a}$ S. Gonzi, ${ }^{56 a, 56 b}$ M. Meschini, ${ }^{56 \mathrm{a}}$ S. Paoletti, ${ }^{56 \mathrm{a}}$ G. Sguazzoni, ${ }^{56 \mathrm{a}}$ A. Tropiano, ${ }^{56 \mathrm{a}, 56 \mathrm{~b}}$ L. Benussi, ${ }^{57}$ S. Bianco, ${ }^{57}$ S. Colafranceschi,${ }^{57, \mathrm{cc}}$ F. Fabbri, ${ }^{57}$ D. Piccolo, ${ }^{57}$ P. Fabbricatore, ${ }^{58 a}$ R. Musenich, ${ }^{58 a}$ S. Tosi, ${ }^{58 a, 58 b}$ A. Benaglia, ${ }^{59 a}$ F. De Guio, ${ }^{59 a, 59 b}$ L. Di Matteo, ${ }^{59 a, 59 b, c}$ S. Fiorendi, ${ }^{59 a, 59 b}$ S. Gennai, ${ }^{59 a, c}$ A. Ghezzi, ${ }^{59 a, 59 b}$ S. Malvezzi, ${ }^{59 a}$ R. A. Manzoni, ${ }^{59 a, 59 b}$ A. Martelli, ${ }^{59 a, 59 b}$ A. Massironi,${ }^{59 a, 59 b}$ D. Menasce, ${ }^{59 a}$ L. Moroni, ${ }^{59 a}$ M. Paganoni, ${ }^{59 a, 59 b}$ D. Pedrini, ${ }^{59 a}$ S. Ragazzi, ${ }^{59 a, 59 b}$ N. Redaelli, ${ }^{59 a}$ S. Sala, ${ }^{59 a}$ T. Tabarelli de Fatis,${ }^{59 a, 59 b}$ S. Buontempo, ${ }^{60 a}$ C. A. Carrillo Montoya, ${ }^{60 a}$ N. Cavallo, ${ }^{60 a, 60 c}$ A. De Cosa,${ }^{60 a, 60 b, c}$ O. Dogangun, ${ }^{60 a, 60 b}$ F. Fabozzi, ${ }^{60 a, 60 c}$ A. O. M. Iorio, ${ }^{60 a, 60 b}$ L. Lista, ${ }^{60 a}$ S. Meola, ${ }^{60 a, 60 d, d d}$ M. Merola, ${ }^{60 a}$ P. Paolucci, ${ }^{60 a, c}$ P. Azzi, ${ }^{61 a}$ N. Bacchetta, ${ }^{61 a, c}$ D. Bisello, ${ }^{61 a, 61 b}$ A. Branca, ${ }^{61 a, 61 b, c}$

R. Carlin, ${ }^{61 \mathrm{a}, 61 \mathrm{~b}}$ P. Checchia, ${ }^{61 \mathrm{a}}$ T. Dorigo, ${ }^{61 \mathrm{a}}$ F. Gasparini, ${ }^{61 \mathrm{a}, 61 \mathrm{~b}}$ A. Gozzelino, ${ }^{61 \mathrm{a}}$ K. Kanishchev, ${ }^{61 \mathrm{a}, 61 \mathrm{c}}$

S. Lacaprara, ${ }^{61 \mathrm{a}}$ I. Lazzizzera, ${ }^{61 \mathrm{a}, 61 \mathrm{c}}$ M. Margoni, ${ }^{61 \mathrm{a}, 61 \mathrm{~b}}$ A. T. Meneguzzo, ${ }^{61 \mathrm{a}, 61 \mathrm{~b}}$ J. Pazzini, ${ }^{61 \mathrm{a}, 61 \mathrm{~b}}$ N. Pozzobon, ${ }^{61 \mathrm{a}, 61 \mathrm{~b}}$ P. Ronchese, ${ }^{61 \mathrm{a}, 61 \mathrm{~b}}$ F. Simonetto, ${ }^{61 \mathrm{a}, 61 \mathrm{~b}}$ E. Torassa, ${ }^{61 \mathrm{a}}$ M. Tosi, ${ }^{61 \mathrm{a}, 61 \mathrm{~b}}$ A. Triossi, ${ }^{61 \mathrm{a}}$ S. Vanini, ${ }^{61 \mathrm{a}, 61 \mathrm{~b}}$ P. Zotto, ${ }^{61 \mathrm{a}, 61 \mathrm{~b}}$ A. Zucchetta, ${ }^{61 \mathrm{a}, 61 \mathrm{~b}}$ G. Zumerle, ${ }^{61 \mathrm{a}, 61 \mathrm{~b}}$ M. Gabusi, ${ }^{62 \mathrm{a}, 62 \mathrm{~b}}$ S. P. Ratti, ${ }^{62 \mathrm{a}, 62 \mathrm{~b}}$ C. Riccardi, ${ }^{62 \mathrm{a}, 62 \mathrm{~b}}$ P. Torre, ${ }^{62 a, 62 \mathrm{~b}}$

P. Vitulo, ${ }^{62 a, 62 b}$ M. Biasini, ${ }^{63 a, 63 b}$ G. M. Bilei, ${ }^{63 a}$ L. Fanò, ${ }^{63 a, 63 b}$ P. Lariccia, ${ }^{63 a, 63 b}$ G. Mantovani, ${ }^{63 a, 63 b}$

M. Menichelli, ${ }^{63 a}$ A. Nappi, ${ }^{63 a, 63 b, a}$ F. Romeo, ${ }^{63 a, 63 b}$ A. Saha, ${ }^{63 a}$ A. Santocchia, ${ }^{63 a, 63 b}$ A. Spiezia, ${ }^{63 a, 63 b}$ S. Taroni, ${ }^{63 a, 63 b}$ P. Azzurri, ${ }^{64 a, 64 c}$ G. Bagliesi, ${ }^{64 a}$ J. Bernardini, ${ }^{64 a}$ T. Boccali, ${ }^{64 a}$ G. Broccolo, ${ }^{64 a, 64 c}$ R. Castaldi ${ }^{64 a}$ R. T. D'Agnolo, ${ }^{64 a, 64 c, c}$ R. Dell'Orso, ${ }^{64 a}$ F. Fiori, ${ }^{64 a, 64 b, c}$ L. Foà,${ }^{64 a, 64 c}$ A. Giassi, ${ }^{64 a}$ A. Kraan, ${ }^{64 a}$ F. Ligabue,${ }^{64 a, 64 c}$ T. Lomtadze ${ }^{64 a}$ L. Martini, ${ }^{64 a, e e}$ A. Messineo ${ }^{64 a, 64 b}$ F. Palla, ${ }^{64 a}$ A. Rizzi, ${ }^{64 a, 64 b}$ A. T. Serban, ${ }^{64 a, f f}$ P. Spagnolo, ${ }^{64 a}$ P. Squillacioti, ${ }^{64 a, c}$ R. Tenchini, ${ }^{64 a}$ G. Tonelli, ${ }^{64 a, 64 b}$ A. Venturi, ${ }^{64 a}$ P. G. Verdini, ${ }^{64 a}$ L. Barone,${ }^{65 a, 65 b}$ F. Cavallari, ${ }^{65 a}$

D. Del Re,${ }^{65 a, 65 b}$ M. Diemoz, ${ }^{65 a}$ C. Fanelli, ${ }^{65 a, 65 b}$ M. Grassi, ${ }^{65 a, 65 b, c}$ E. Longo, ${ }^{65 a, 65 b}$ P. Meridiani, ${ }^{65 a, c}$

F. Micheli, ${ }^{65 a, 65 b}$ S. Nourbakhsh ${ }^{65 a, 65 b}$ G. Organtini, ${ }^{65 a, 65 b}$ R. Paramatti, ${ }^{65 a}$ S. Rahatlou, ${ }^{65 a, 65 b}$ M. Sigamani, ${ }^{65 a}$

L. Soffi, ${ }^{65 a, 65 b}$ N. Amapane,${ }^{66 a, 66 b}$ R. Arcidiacono, ${ }^{66 a, 66 c}$ S. Argiro, ${ }^{66 a, 66 b}$ M. Arneodo,${ }^{66 a, 66 c}$ C. Biino, ${ }^{66 a}$

N. Cartiglia, ${ }^{66 \mathrm{a}}$ S. Casasso, ${ }^{66 a, 66 \mathrm{~b}}$ M. Costa, ${ }^{66 \mathrm{a}, 66 \mathrm{~b}}$ N. Demaria, ${ }^{66 \mathrm{a}}$ C. Mariotti ${ }^{66 \mathrm{a}, \mathrm{c}}$ S. Maselli, ${ }^{66 \mathrm{a}}$ E. Migliore, ${ }^{66 a, 66 b}$ V. Monaco, ${ }^{66 a, 66 b}$ M. Musich, ${ }^{66 a, c}$ M. M. Obertino, ${ }^{66 a, 66 c}$ N. Pastrone ${ }^{66 a}$ M. Pelliccioni, ${ }^{66 a}$ A. Potenza, ${ }^{66 a, 66 b}$ A. Romero, ${ }^{66 a, 66 b}$ M. Ruspa,${ }^{66 a, 66 c}$ R. Sacchi, ${ }^{66 a, 66 b}$ A. Solano, ${ }^{66 a, 66 b}$ A. Staiano, ${ }^{66 a}$ S. Belforte, ${ }^{67 a}$ V. Candelise, ${ }^{67 a, 67 b}$ M. Casarsa, ${ }^{67 a}$ F. Cossutti, ${ }^{67 a}$ G. Della Ricca, ${ }^{67 a, 67 b}$ B. Gobbo, ${ }^{67 a}$ M. Marone,${ }^{67 a, 67 b, c}$ 
D. Montanino, ${ }^{67 a, 67 b, c}$ A. Penzo, ${ }^{67 a}$ A. Schizzi, ${ }^{67 a, 67 b}$ T. Y. Kim, ${ }^{68}$ S. K. Nam,${ }^{68}$ S. Chang, ${ }^{69}$ D. H. Kim,${ }^{69}$ G. N. Kim, ${ }^{69}$ D. J. Kong, ${ }^{69}$ H. Park, ${ }^{69}$ D. C. Son, ${ }^{69}$ T. Son, ${ }^{69}$ J. Y. Kim, ${ }^{70}$ Zero J. Kim, ${ }^{70}$ S. Song, ${ }^{70}$ S. Choi, ${ }^{71}$ D. Gyun, ${ }^{71}$ B. Hong,${ }^{71}$ M. Jo, ${ }^{71}$ H. Kim, ${ }^{71}$ T. J. Kim, ${ }^{71}$ K. S. Lee, ${ }^{71}$ D. H. Moon,${ }^{71}$ S. K. Park, ${ }^{71}$ Y. Roh, ${ }^{71}$ M. Choi, ${ }^{72}$ J. H. Kim,${ }^{72}$ C. Park, ${ }^{72}$ I. C. Park, ${ }^{72}$ S. Park, ${ }^{72}$ G. Ryu ${ }^{72}$ Y. Choi, ${ }^{73}$ Y. K. Choi ${ }^{73}$ J. Goh,${ }^{73}$ M. S. Kim, ${ }^{73}$ E. Kwon, ${ }^{73}$ B. Lee, ${ }^{73}$ J. Lee, ${ }^{73}$ S. Lee, ${ }^{73}$ H. Seo, ${ }^{73}$ I. Yu, ${ }^{73}$ M. J. Bilinskas, ${ }^{74}$ I. Grigelionis, ${ }^{74}$ M. Janulis, ${ }^{74}$ A. Juodagalvis, ${ }^{74}$ H. Castilla-Valdez, ${ }^{75}$ E. De La Cruz-Burelo, ${ }^{75}$ I. Heredia-de La Cruz ${ }^{75}$ R. Lopez-Fernandez, ${ }^{75}$ J. Martínez-Ortega, ${ }^{75}$ A. Sanchez-Hernandez, ${ }^{75}$ L. M. Villasenor-Cendejas, ${ }^{75}$ S. Carrillo Moreno, ${ }^{76}$ F. Vazquez Valencia, ${ }^{76}$

H. A. Salazar Ibarguen, ${ }^{77}$ E. Casimiro Linares, ${ }^{78}$ A. Morelos Pineda, ${ }^{78}$ M. A. Reyes-Santos ${ }^{78}$ D. Krofcheck,${ }^{79}$ A. J. Bell, ${ }^{80}$ P. H. Butler, ${ }^{80}$ R. Doesburg, ${ }^{80}$ S. Reucroft, ${ }^{80}$ H. Silverwood, ${ }^{80}$ M. Ahmad, ${ }^{81}$ M. I. Asghar, ${ }^{81}$ J. Butt, ${ }^{81}$ H. R. Hoorani, ${ }^{81}$ S. Khalid, ${ }^{81}$ W. A. Khan,${ }^{81}$ T. Khurshid, ${ }^{81}$ S. Qazi, ${ }^{81}$ M. A. Shah, ${ }^{81}$ M. Shoaib ${ }^{81}$ H. Bialkowska, ${ }^{82}$ B. Boimska, ${ }^{82}$ T. Frueboes, ${ }^{82}$ M. Górski, ${ }^{82}$ M. Kazana,${ }^{82} \mathrm{~K}$. Nawrocki, ${ }^{82} \mathrm{~K}$. Romanowska-Rybinska, ${ }^{82} \mathrm{M}$. Szleper, ${ }^{82}$ G. Wrochna ${ }^{82}$ P. Zalewski, ${ }^{82}$ G. Brona,${ }^{83}$ K. Bunkowski, ${ }^{83}$ M. Cwiok, ${ }^{83}$ W. Dominik, ${ }^{83}$ K. Doroba,${ }^{83}$ A. Kalinowski, ${ }^{83}$ M. Konecki, ${ }^{83}$ J. Krolikowski, ${ }^{83}$ M. Misiura,${ }^{83}$ N. Almeida ${ }^{84}$ P. Bargassa ${ }^{84}$ A. David ${ }^{84}$ P. Faccioli, ${ }^{84}$ P. G. Ferreira Parracho,${ }^{84}$ M. Gallinaro, ${ }^{84}$ J. Seixas, ${ }^{84}$ J. Varela,${ }^{84}$ P. Vischia,${ }^{84}$ P. Bunin,${ }^{85}$ M. Gavrilenko, ${ }^{85}$ I. Golutvin, ${ }^{85}$ I. Gorbunov, ${ }^{85}$ A. Kamenev, ${ }^{85}$ V. Karjavin,${ }^{85}$ G. Kozlov, ${ }^{85}$ A. Lanev, ${ }^{85}$ A. Malakhov ${ }^{85}$ P. Moisenz, ${ }^{85}$ V. Palichik, ${ }^{85}$ V. Perelygin, ${ }^{85}$ M. Savina,${ }^{85}$ S. Shmatov, ${ }^{85}$ V. Smirnov, ${ }^{85}$ A. Volodko, ${ }^{85}$ A. Zarubin, ${ }^{85}$ S. Evstyukhin, ${ }^{86}$ V. Golovtsov ${ }^{86}$ Y. Ivanov, ${ }^{86}$ V. Kim, ${ }^{86}$ P. Levchenko, ${ }^{86}$ V. Murzin, ${ }^{86}$ V. Oreshkin, ${ }^{86}$ I. Smirnov, ${ }^{86}$ V. Sulimov, ${ }^{86}$ L. Uvarov, ${ }^{86}$ S. Vavilov, ${ }^{86}$ A. Vorobyev,${ }^{86}$ An. Vorobyev, ${ }^{86}$ Yu. Andreev, ${ }^{87}$ A. Dermenev,${ }^{87}$ S. Gninenko, ${ }^{87}$ N. Golubev, ${ }^{87}$ M. Kirsanov, ${ }^{87}$ N. Krasnikov, ${ }^{87}$ V. Matveev, ${ }^{87}$ A. Pashenkov, ${ }^{87}$ D. Tlisov, ${ }^{87}$ A. Toropin, ${ }^{87}$ V. Epshteyn,${ }^{88}$ M. Erofeeva, ${ }^{88}$ V. Gavrilov, ${ }^{88}$ M. Kossov, ${ }^{88}$ N. Lychkovskaya, ${ }^{88}$ V. Popov, ${ }^{88}$ G. Safronov, ${ }^{88}$ S. Semenov, ${ }^{88}$ I. Shreyber, ${ }^{88}$ V. Stolin, ${ }^{88}$ E. Vlasov, ${ }^{88}$ A. Zhokin, ${ }^{88}$ A. Belyaev, ${ }^{89}$ E. Boos,${ }^{89}$ M. Dubinin, ${ }^{89, e}$ L. Dudko, ${ }^{89}$ A. Ershov, ${ }^{89}$ A. Gribushin, ${ }^{89}$ V. Klyukhin, ${ }^{89}$ O. Kodolova, ${ }^{89}$ I. Lokhtin,${ }^{89}$ A. Markina, ${ }^{89}$ S. Obraztsov ${ }^{89}$ M. Perfilov ${ }^{89}$ S. Petrushanko, ${ }^{89}$ A. Popov,${ }^{89}$ L. Sarycheva,${ }^{89, a}$ V. Savrin, ${ }^{89}$ A. Snigirev,${ }^{89}$ V. Andreev,${ }^{90}$ M. Azarkin, ${ }^{90}$ I. Dremin,,${ }^{90}$ M. Kirakosyan, ${ }^{90}$ A. Leonidov, ${ }^{90}$ G. Mesyats, ${ }^{90}$ S. V. Rusakov, ${ }^{90}$ A. Vinogradov,${ }^{90}$ I. Azhgirey, ${ }^{91}$ I. Bayshev, ${ }^{91}$ S. Bitioukov, ${ }^{91}$ V. Grishin, ${ }^{91, \mathrm{c}}$ V. Kachanov, ${ }^{91}$ D. Konstantinov, ${ }^{91}$ V. Krychkine, ${ }^{91}$ V. Petrov, ${ }^{91}$ R. Ryutin, ${ }^{91}$ A. Sobol, ${ }^{91}$ L. Tourtchanovitch, ${ }^{91}$ S. Troshin, ${ }^{91}$ N. Tyurin, ${ }^{91}$ A. Uzunian, ${ }^{91}$ A. Volkov, ${ }^{91}$ P. Adzic, ${ }^{92, g g}$ M. Djordjevic, ${ }^{92}$ M. Ekmedzic, ${ }^{92}$ D. Krpic,${ }^{92, g g}$ J. Milosevic, ${ }^{92}$ M. Aguilar-Benitez, ${ }^{93}$ J. Alcaraz Maestre, ${ }^{93}$ P. Arce, ${ }^{93}$ C. Battilana,${ }^{93}$ E. Calvo,${ }^{93}$ M. Cerrada, ${ }^{93}$ M. Chamizo Llatas, ${ }^{93}$ N. Colino, ${ }^{93}$ B. De La Cruz, ${ }^{93}$ A. Delgado Peris, ${ }^{93}$ D. Domínguez Vázquez, ${ }^{93}$ C. Fernandez Bedoya ${ }^{93}$ J. P. Fernández Ramos, ${ }^{93}$ A. Ferrando, ${ }^{93}$ J. Flix, ${ }^{93}$ M. C. Fouz, ${ }^{93}$ P. Garcia-Abia, ${ }^{93}$ O. Gonzalez Lopez, ${ }^{93}$ S. Goy Lopez,${ }^{93}$ J. M. Hernandez,${ }^{93}$ M. I. Josa,${ }^{93}$ G. Merino, ${ }^{93}$ J. Puerta Pelayo,${ }^{93}$ A. Quintario Olmeda,${ }^{93}$ I. Redondo, ${ }^{93}$ L. Romero, ${ }^{93}$ J. Santaolalla, ${ }^{93}$ M. S. Soares, ${ }^{93}$ C. Willmott, ${ }^{93}$ C. Albajar,${ }^{94}$ G. Codispoti, ${ }^{94}$ J. F. de Trocóniz,${ }^{94}$ H. Brun, ${ }^{95}$ J. Cuevas, ${ }^{95}$ J. Fernandez Menendez, ${ }^{95}$ S. Folgueras, ${ }^{95}$ I. Gonzalez Caballero, ${ }^{95}$ L. Lloret Iglesias, ${ }^{95}$ J. Piedra Gomez,${ }^{95}$ J. A. Brochero Cifuentes, ${ }^{96}$ I. J. Cabrillo, ${ }^{96}$ A. Calderon, ${ }^{96}$ S. H. Chuang,,${ }^{96}$ J. Duarte Campderros, ${ }^{96}$ M. Felcini, ${ }^{96, \text { hh }}$ M. Fernandez, ${ }^{96}$ G. Gomez,${ }^{96}$ J. Gonzalez Sanchez, ${ }^{96}$ A. Graziano, ${ }^{96}$ C. Jorda,${ }^{96}$ A. Lopez Virto, ${ }^{96}$ J. Marco, ${ }^{96}$ R. Marco, ${ }^{96}$ C. Martinez Rivero, ${ }^{96}$ F. Matorras, ${ }^{96}$ F. J. Munoz Sanchez, ${ }^{96}$ T. Rodrigo, ${ }^{96}$ A. Y. Rodríguez-Marrero, ${ }^{96}$ A. Ruiz-Jimeno ${ }^{96}$ L. Scodellaro, ${ }^{96}$ I. Vila ${ }^{96}$ R. Vilar Cortabitarte, ${ }^{96}$ D. Abbaneo, ${ }^{97}$ E. Auffray,${ }^{97}$ G. Auzinger,${ }^{97}$ M. Bachtis, ${ }^{97}$ P. Baillon, ${ }^{97}$ A. H. Ball, ${ }^{97}$ D. Barney, ${ }^{97}$ J. F. Benitez,${ }^{97}$ C. Bernet,${ }^{97, f}$ G. Bianchi, ${ }^{97}$ P. Bloch, ${ }^{97}$ A. Bocci, ${ }^{97}$ A. Bonato, ${ }^{97}$ C. Botta, ${ }^{97}$ H. Breuker, ${ }^{97}$ T. Camporesi, ${ }^{97}$ G. Cerminara, ${ }^{97}$ T. Christiansen, ${ }^{97}$ J. A. Coarasa Perez,${ }^{97}$ D. D'Enterria, ${ }^{97}$ A. Dabrowski, ${ }^{97}$ A. De Roeck,${ }^{97}$ S. Di Guida, ${ }^{97}$ M. Dobson,,${ }^{97}$ N. Dupont-Sagorin, ${ }^{97}$ A. Elliott-Peisert, ${ }^{97}$ B. Frisch, ${ }^{97}$ W. Funk,${ }^{97}$ G. Georgiou, ${ }^{97}$ M. Giffels,,${ }^{97}$ D. Gigi,${ }^{97}$ K. Gill, ${ }^{97}$ D. Giordano,${ }^{97}$ M. Girone, ${ }^{97}$ M. Giunta,${ }^{97}$ F. Glege,${ }^{97}$ R. Gomez-Reino Garrido, ${ }^{97}$ P. Govoni,${ }^{97}$ S. Gowdy, ${ }^{97}$ R. Guida, ${ }^{97}$ S. Gundacker, ${ }^{97}$ J. Hammer,${ }^{97}$ M. Hansen, ${ }^{97}$ P. Harris,${ }^{97}$ C. Hartl, ${ }^{97}$ J. Harvey, ${ }^{97}$ B. Hegner, ${ }^{97}$ A. Hinzmann, ${ }^{97}$ V. Innocente, ${ }^{97}$ P. Janot, ${ }^{97}$ K. Kaadze, ${ }^{97}$ E. Karavakis, ${ }^{97}$ K. Kousouris, ${ }^{97}$ P. Lecoq ${ }^{97}$ Y.-J. Lee,${ }^{97}$ P. Lenzi, ${ }^{97}$ C. Lourenço, ${ }^{97}$ N. Magini,${ }^{97}$ T. Mäki,${ }^{97}$ M. Malberti, ${ }^{97}$ L. Malgeri, ${ }^{97}$ M. Mannelli, ${ }^{97}$ L. Masetti, ${ }^{97}$ F. Meijers, ${ }^{97}$ S. Mersi, ${ }^{97}$ E. Meschi,${ }^{97}$ R. Moser,${ }^{97}$ M. U. Mozer, ${ }^{97}$ M. Mulders, ${ }^{97}$ P. Musella,${ }^{97}$ E. Nesvold, ${ }^{97}$ L. Orsini, ${ }^{97}$ E. Palencia Cortezon, ${ }^{97}$ E. Perez, ${ }^{97}$ L. Perrozzi, ${ }^{97}$ A. Petrilli, ${ }^{97}$ A. Pfeiffer, ${ }^{97}$ M. Pierini, ${ }^{97}$ M. Pimiä, ${ }^{97}$ D. Piparo, ${ }^{97}$ G. Polese,${ }^{97}$ L. Quertenmont,${ }^{97}$ A. Racz,${ }^{97}$ W. Reece,,${ }^{97}$ J. Rodrigues Antunes,${ }^{97}$ G. Rolandi, ${ }^{97, \text { ii }}$

C. Rovelli, ${ }^{97, j j}$ M. Rovere, ${ }^{97}$ H. Sakulin, ${ }^{97}$ F. Santanastasio, ${ }^{97}$ C. Schäfer, ${ }^{97}$ C. Schwick, ${ }^{97}$ I. Segoni, ${ }^{97}$ S. Sekmen, ${ }^{97}$ A. Sharma,${ }^{97}$ P. Siegrist,${ }^{97}$ P. Silva, ${ }^{97}$ M. Simon, ${ }^{97}$ P. Sphicas,,${ }^{97, k k}$ D. Spiga,${ }^{97}$ A. Tsirou,${ }^{97}$ G. I. Veres, ${ }^{97, u}$ J. R. Vlimant, ${ }^{97}$ H. K. Wöhri, ${ }^{97}$ S. D. Worm,,${ }^{97,11}$ W. D. Zeuner, ${ }^{97}$ W. Bertl, ${ }^{98}$ K. Deiters,${ }^{98}$ W. Erdmann, ${ }^{98}$ 
K. Gabathuler, ${ }^{98}$ R. Horisberger, ${ }^{98}$ Q. Ingram, ${ }^{98}$ H. C. Kaestli, ${ }^{98}$ S. König, ${ }^{98}$ D. Kotlinski, ${ }^{98}$ U. Langenegger, ${ }^{98}$

F. Meier ${ }^{98}$ D. Renker, ${ }^{98}$ T. Rohe, ${ }^{98}$ L. Bäni,${ }^{99}$ P. Bortignon, ${ }^{99}$ M. A. Buchmann, ${ }^{99}$ B. Casal, ${ }^{99}$ N. Chanon, ${ }^{99}$ A. Deisher, ${ }^{99}$ G. Dissertori, ${ }^{99}$ M. Dittmar, ${ }^{99}$ M. Donegà, ${ }^{99}$ M. Dünser, ${ }^{99}$ P. Eller, ${ }^{99}$ J. Eugster, ${ }^{99}$ K. Freudenreich,${ }^{99}$ C. Grab, ${ }^{99}$ D. Hits, ${ }^{99}$ P. Lecomte, ${ }^{99}$ W. Lustermann, ${ }^{99}$ A. C. Marini, ${ }^{99}$ P. Martinez Ruiz del Arbol, ${ }^{99}$ N. Mohr, ${ }^{99}$ F. Moortgat, ${ }^{99}$ C. Nägeli, ${ }^{99, \mathrm{~mm}}$ P. Nef, ${ }^{99}$ F. Nessi-Tedaldi, ${ }^{99}$ F. Pandolfi, ${ }^{99}$ L. Pape,${ }^{99}$ F. Pauss, ${ }^{99}$ M. Peruzzi,${ }^{99}$ F. J. Ronga, ${ }^{99}$ M. Rossini, ${ }^{99}$ L. Sala, ${ }^{99}$ A. K. Sanchez, ${ }^{99}$ A. Starodumov, ${ }^{99}{ }^{\text {nn }}$ B. Stieger, ${ }^{99}$ M. Takahashi, ${ }^{99}$ L. Tauscher, ${ }^{99, a}$ A. Thea, ${ }^{99}$ K. Theofilatos, ${ }^{99}$ D. Treille, ${ }^{99}$ C. Urscheler, ${ }^{99}$ R. Wallny, ${ }^{99}$ H. A. Weber, ${ }^{99}$ L. Wehrli, ${ }^{99}$ C. Amsler, ${ }^{100, \text { oo }}$ V. Chiochia, ${ }^{100}$ S. De Visscher, ${ }^{100}$ C. Favaro, ${ }^{100}$ M. Ivova Rikova, ${ }^{100}$ B. Kilminster,${ }^{100}$ B. Millan Mejias, ${ }^{100}$ P. Otiougova, ${ }^{100}$ P. Robmann, ${ }^{100}$ H. Snoek, ${ }^{100}$ S. Tupputi, ${ }^{100}$ M. Verzetti, ${ }^{100}$ Y. H. Chang, ${ }^{101}$ K. H. Chen,,${ }^{101}$ C. Ferro, ${ }^{101}$ C. M. Kuo, ${ }^{101}$ S. W. Li, ${ }^{101}$ W. Lin, ${ }^{101}$ Y. J. Lu, ${ }^{101}$ A. P. Singh, ${ }^{101}$ R. Volpe, ${ }^{101}$ S. S. Yu, ${ }^{101}$ P. Bartalini, ${ }^{102}$ P. Chang, ${ }^{102}$ Y. H. Chang, ${ }^{102}$ Y. W. Chang,,${ }^{102}$ Y. Chao, ${ }^{102}$ K. F. Chen, ${ }^{102}$ C. Dietz, ${ }^{102}$ U. Grundler, ${ }^{102}$ W.-S. Hou, ${ }^{102}$ Y. Hsiung, ${ }^{102}$ K. Y. Kao, ${ }^{102}$ Y. J. Lei,${ }^{102}$ R.-S. Lu, ${ }^{102}$ D. Majumder, ${ }^{102}$ E. Petrakou, ${ }^{102}$ X. Shi, ${ }^{102}$ J. G. Shiu, ${ }^{102}$ Y. M. Tzeng, ${ }^{102}$ X. Wan,${ }^{102}$ M. Wang,,${ }^{102}$ B. Asavapibhop, ${ }^{103}$ N. Srimanobhas, ${ }^{103}$ A. Adiguzel, ${ }^{104}$ M. N. Bakirci, ${ }^{104, p p}$ S. Cerci, ${ }^{104, q 9}$ C. Dozen, ${ }^{104}$ I. Dumanoglu, ${ }^{104}$ E. Eskut, ${ }^{104}$ S. Girgis, ${ }^{104}$ G. Gokbulut,${ }^{104}$ E. Gurpinar, ${ }^{104}$ I. Hos, ${ }^{104}$ E. E. Kangal, ${ }^{104}$ T. Karaman, ${ }^{104}$ G. Karapinar, ${ }^{104, r r}$ A. Kayis Topaksu, ${ }^{104}$ G. Onengut, ${ }^{104}$ K. Ozdemir, ${ }^{104}$ S. Ozturk, ${ }^{104, \mathrm{ss}}$ A. Polatoz, ${ }^{104}$ K. Sogut, ${ }^{104, \mathrm{tt}}$ D. Sunar Cerci, ${ }^{104, \mathrm{qq}}$ B. Tali, ${ }^{104, \mathrm{qq}}$ H. Topakli, ${ }^{104, \mathrm{pp}}$ L. N. Vergili, ${ }^{104}$ M. Vergili, ${ }^{104}$ I. V. Akin, ${ }^{105}$ T. Aliev, ${ }^{105}$ B. Bilin, ${ }^{105}$ S. Bilmis, ${ }^{105}$ M. Deniz, ${ }^{105}$ H. Gamsizkan, ${ }^{105}$ A. M. Guler, ${ }^{105}$ K. Ocalan, ${ }^{105}$ A. Ozpineci, ${ }^{105}$ M. Serin, ${ }^{105}$ R. Sever, ${ }^{105}$ U. E. Surat, ${ }^{105}$ M. Yalvac, ${ }^{105}$ E. Yildirim, ${ }^{105}$ M. Zeyrek, ${ }^{105}$ E. Gülmez, ${ }^{106}$ B. Isildak, ${ }^{106, \text { uu }}$ M. Kaya, ${ }^{106, v v}$ O. Kaya, ${ }^{106, v v}$ S. Ozkorucuklu, ${ }^{106, w w}$ N. Sonmez, ${ }^{106, x x}$

K. Cankocak, ${ }^{107}$ L. Levchuk, ${ }^{108}$ J. J. Brooke, ${ }^{109}$ E. Clement, ${ }^{109}$ D. Cussans, ${ }^{109}$ H. Flacher, ${ }^{109}$ R. Frazier, ${ }^{109}$ J. Goldstein, ${ }^{109}$ M. Grimes, ${ }^{109}$ G. P. Heath, ${ }^{109}$ H. F. Heath, ${ }^{109}$ L. Kreczko, ${ }^{109}$ S. Metson, ${ }^{109}$ D. M. Newbold, ${ }^{109,11}$

K. Nirunpong, ${ }^{109}$ A. Poll, ${ }^{109}$ S. Senkin, ${ }^{109}$ V. J. Smith, ${ }^{109}$ T. Williams, ${ }^{109}$ L. Basso, ${ }^{110, y y}$ K. W. Bell,,${ }^{110}$ A. Belyaev, ${ }^{110, y y}$ C. Brew, ${ }^{110}$ R. M. Brown, ${ }^{110}$ D. J. A. Cockerill, ${ }^{110}$ J. A. Coughlan, ${ }^{110}$ K. Harder, ${ }^{110}$ S. Harper, ${ }^{110}$ J. Jackson, ${ }^{110}$ B. W. Kennedy, ${ }^{10}$ E. Olaiya, ${ }^{110}$ D. Petyt, ${ }^{110}$ B. C. Radburn-Smith, ${ }^{110}$

C. H. Shepherd-Themistocleous, ${ }^{110}$ I. R. Tomalin, ${ }^{110}$ W. J. Womersley, ${ }^{110}$ R. Bainbridge, ${ }^{111}$ G. Ball,,${ }^{11}$ R. Beuselinck, ${ }^{111}$ O. Buchmuller, ${ }^{11}$ D. Colling, ${ }^{111}$ N. Cripps, ${ }^{111}$ M. Cutajar, ${ }^{111}$ P. Dauncey, ${ }^{111}$ G. Davies, ${ }^{111}$ M. Della Negra, ${ }^{111}$ W. Ferguson, ${ }^{111}$ J. Fulcher, ${ }^{111}$ D. Futyan, ${ }^{111}$ A. Gilbert, ${ }^{111}$ A. Guneratne Bryer, ${ }^{111}$ G. Hall, ${ }^{111}$ Z. Hatherell, ${ }^{111}$ J. Hays, ${ }^{111}$ G. Iles, ${ }^{111}$ M. Jarvis, ${ }^{111}$ G. Karapostoli, ${ }^{111}$ L. Lyons, ${ }^{111}$ A.-M. Magnan, ${ }^{111}$

J. Marrouche, ${ }^{111}$ B. Mathias, ${ }^{111}$ R. Nandi, ${ }^{111}$ J. Nash, ${ }^{11}$ A. Nikitenko, ${ }^{111, \text { nn }}$ J. Pela, ${ }^{111}$ M. Pesaresi, ${ }^{111}$ K. Petridis,${ }^{111}$ M. Pioppi, ${ }^{111, z z}$ D. M. Raymond, ${ }^{111}$ S. Rogerson, ${ }^{111}$ A. Rose, ${ }^{111}$ M. J. Ryan, ${ }^{111}$ C. Seez, ${ }^{111}$ P. Sharp, ${ }^{11, a}$

A. Sparrow, ${ }^{111}$ M. Stoye, ${ }^{111}$ A. Tapper, ${ }^{111}$ M. Vazquez Acosta, ${ }^{111}$ T. Virdee,${ }^{111}$ S. Wakefield, ${ }^{111}$ N. Wardle,${ }^{111}$ T. Whyntie, ${ }^{111}$ M. Chadwick, ${ }^{112}$ J. E. Cole, ${ }^{112}$ P. R. Hobson, ${ }^{112}$ A. Khan, ${ }^{112}$ P. Kyberd, ${ }^{112}$ D. Leggat,${ }^{112}$ D. Leslie, ${ }^{112}$ W. Martin, ${ }^{12}$ I. D. Reid, ${ }^{112}$ P. Symonds, ${ }^{12}$ L. Teodorescu, ${ }^{112}$ M. Turner, ${ }^{112}$ K. Hatakeyama, ${ }^{13}$ H. Liu, ${ }^{113}$ T. Scarborough, ${ }^{113}$ O. Charaf, ${ }^{114}$ C. Henderson, ${ }^{114}$ P. Rumerio, ${ }^{114}$ A. Avetisyan, ${ }^{115}$ T. Bose,,${ }^{115}$ C. Fantasia, ${ }^{115}$ A. Heister, ${ }^{115}$ J. St. John, ${ }^{115}$ P. Lawson, ${ }^{15}$ D. Lazic,,${ }^{15}$ J. Rohlf, ${ }^{115}$ D. Sperka, ${ }^{115}$ L. Sulak, ${ }^{115}$ J. Alimena, ${ }^{116}$

S. Bhattacharya, ${ }^{116}$ G. Christopher, ${ }^{116}$ D. Cutts, ${ }^{116}$ Z. Demiragli, ${ }^{116}$ A. Ferapontov, ${ }^{116}$ A. Garabedian, ${ }^{116}$ U. Heintz, ${ }^{116}$ S. Jabeen, ${ }^{116}$ G. Kukartsev, ${ }^{116}$ E. Laird, ${ }^{116}$ G. Landsberg, ${ }^{116}$ M. Luk, ${ }^{116}$ M. Narain, ${ }^{116}$ D. Nguyen, ${ }^{116}$ M. Segala, ${ }^{16}$ T. Sinthuprasith, ${ }^{16}$ T. Speer, ${ }^{16}$ R. Breedon, ${ }^{17}$ G. Breto, ${ }^{117}$ M. Calderon De La Barca Sanchez, ${ }^{117}$ S. Chauhan, ${ }^{117}$ M. Chertok, ${ }^{117}$ J. Conway, ${ }^{117}$ R. Conway, ${ }^{117}$ P. T. Cox,${ }^{117}$ J. Dolen, ${ }^{117}$ R. Erbacher, ${ }^{117}$ M. Gardner, ${ }^{117}$ R. Houtz, ${ }^{117}$ W. Ko, ${ }^{117}$ A. Kopecky, ${ }^{117}$ R. Lander, ${ }^{117}$ O. Mall, ${ }^{117}$ T. Miceli, ${ }^{117}$ D. Pellett, ${ }^{117}$ F. Ricci-Tam, ${ }^{117}$ B. Rutherford, ${ }^{117}$ M. Searle, ${ }^{117}$ J. Smith, ${ }^{117}$ M. Squires, ${ }^{117}$ M. Tripathi, ${ }^{117}$ R. Vasquez Sierra, ${ }^{117}$ R. Yohay, ${ }^{117}$ V. Andreev, ${ }^{118}$ D. Cline, ${ }^{118}$ R. Cousins, ${ }^{118}$ J. Duris, ${ }^{118}$ S. Erhan, ${ }^{118}$ P. Everaerts, ${ }^{118}$ C. Farrell, ${ }^{118}$ J. Hauser, ${ }^{118}$ M. Ignatenko, ${ }^{118}$ C. Jarvis, ${ }^{118}$ G. Rakness, ${ }^{118}$ P. Schlein, ${ }^{18, a}$ P. Traczyk, ${ }^{118}$ V. Valuev, ${ }^{18}$ M. Weber, ${ }^{18}$ J. Babb, ${ }^{119}$ R. Clare, ${ }^{119}$ M. E. Dinardo, ${ }^{119}$ J. Ellison, ${ }^{119}$ J. W. Gary, ${ }^{119}$ F. Giordano, ${ }^{119}$ G. Hanson, ${ }^{119}$ H. Liu, ${ }^{119}$ O. R. Long, ${ }^{119}$ A. Luthra, ${ }^{119}$ H. Nguyen, ${ }^{119}$ S. Paramesvaran, ${ }^{119}$ J. Sturdy, ${ }^{119}$ S. Sumowidagdo, ${ }^{119}$ R. Wilken,${ }^{119}$ S. Wimpenny, ${ }^{119}$ W. Andrews, ${ }^{120}$ J. G. Branson, ${ }^{120}$ G. B. Cerati, ${ }^{120}$ S. Cittolin, ${ }^{120}$ D. Evans, ${ }^{120}$ A. Holzner, ${ }^{120}$ R. Kelley, ${ }^{120}$ M. Lebourgeois, ${ }^{120}$ J. Letts, ${ }^{120}$ I. Macneill, ${ }^{120}$ B. Mangano, ${ }^{120}$ S. Padhi, ${ }^{120}$ C. Palmer, ${ }^{120}$ G. Petrucciani, ${ }^{120}$ M. Pieri,,${ }^{120}$ M. Sani, ${ }^{120}$ V. Sharma, ${ }^{120}$ S. Simon, ${ }^{120}$ E. Sudano, ${ }^{120}$ M. Tadel, ${ }^{120}$ Y. Tu, ${ }^{120}$ A. Vartak,,${ }^{120}$

S. Wasserbaech, ${ }^{120 \text {,aaa }}$ F. Würthwein, ${ }^{120}$ A. Yagil, ${ }^{120}$ J. Yoo, ${ }^{120}$ D. Barge,${ }^{121}$ R. Bellan, ${ }^{121}$ C. Campagnari, ${ }^{121}$ M. D'Alfonso, ${ }^{121}$ T. Danielson, ${ }^{121}$ K. Flowers, ${ }^{121}$ P. Geffert, ${ }^{121}$ F. Golf, ${ }^{121}$ J. Incandela, ${ }^{121}$ C. Justus, ${ }^{121}$ P. Kalavase, ${ }^{121}$ D. Kovalskyi, ${ }^{121}$ V. Krutelyov, ${ }^{121}$ S. Lowette, ${ }^{121}$ R. Magaña Villalba, ${ }^{121}$ N. Mccoll, ${ }^{121}$ V. Pavlunin, ${ }^{121}$ 
J. Ribnik, ${ }^{121}$ J. Richman, ${ }^{121}$ R. Rossin, ${ }^{121}$ D. Stuart, ${ }^{121}$ W. To, ${ }^{121}$ C. West, ${ }^{121}$ A. Apresyan, ${ }^{122}$ A. Bornheim, ${ }^{122}$ Y. Chen, ${ }^{122}$ E. Di Marco, ${ }^{122}$ J. Duarte, ${ }^{122}$ M. Gataullin, ${ }^{122}$ Y. Ma,${ }^{122}$ A. Mott,,${ }^{122}$ H. B. Newman, ${ }^{122}$ C. Rogan, ${ }^{122}$ M. Spiropulu, ${ }^{122}$ V. Timciuc, ${ }^{122}$ J. Veverka, ${ }^{122}$ R. Wilkinson, ${ }^{122}$ S. Xie, ${ }^{122}$ Y. Yang, ${ }^{122}$ R. Y. Zhu, ${ }^{122}$ V. Azzolini, ${ }^{123}$ A. Calamba, ${ }^{123}$ R. Carroll, ${ }^{123}$ T. Ferguson, ${ }^{123}$ Y. Iiyama, ${ }^{123}$ D. W. Jang, ${ }^{123}$ Y. F. Liu, ${ }^{123}$ M. Paulini, ${ }^{123}$ H. Vogel, ${ }^{123}$ I. Vorobiev, ${ }^{123}$ J. P. Cumalat, ${ }^{124}$ B. R. Drell, ${ }^{124}$ W. T. Ford, ${ }^{124}$ A. Gaz, ${ }^{124}$ E. Luiggi Lopez, ${ }^{124}$ J. G. Smith, ${ }^{124}$

K. Stenson, ${ }^{124}$ K. A. Ulmer, ${ }^{124}$ S. R. Wagner, ${ }^{124}$ J. Alexander, ${ }^{125}$ A. Chatterjee, ${ }^{125}$ N. Eggert, ${ }^{125}$ L. K. Gibbons, ${ }^{125}$ B. Heltsley, ${ }^{125}$ W. Hopkins, ${ }^{125}$ A. Khukhunaishvili, ${ }^{125}$ B. Kreis,${ }^{125}$ N. Mirman ${ }^{125}$ G. Nicolas Kaufman, ${ }^{125}$

J. R. Patterson, ${ }^{125}$ A. Ryd, ${ }^{125}$ E. Salvati, ${ }^{125}$ W. Sun, ${ }^{125}$ W. D. Teo, ${ }^{125}$ J. Thom,${ }^{125}$ J. Thompson,,${ }^{125}$ J. Tucker, ${ }^{125}$ J. Vaughan, ${ }^{125}$ Y. Weng, ${ }^{125}$ L. Winstrom, ${ }^{125}$ P. Wittich, ${ }^{125}$ D. Winn,,${ }^{126}$ S. Abdullin, ${ }^{127}$ M. Albrow, ${ }^{127}$ J. Anderson, ${ }^{127}$ L. A. T. Bauerdick, ${ }^{127}$ A. Beretvas, ${ }^{127}$ J. Berryhill, ${ }^{127}$ P. C. Bhat, ${ }^{127}$ K. Burkett, ${ }^{127}$ J. N. Butler, ${ }^{127}$ V. Chetluru, ${ }^{127}$ H. W. K. Cheung, ${ }^{127}$ F. Chlebana, ${ }^{127}$ V. D. Elvira, ${ }^{127}$ I. Fisk, ${ }^{127}$ J. Freeman, ${ }^{127}$ Y. Gao, ${ }^{127}$ D. Green, ${ }^{127}$ O. Gutsche,${ }^{127}$ J. Hanlon, ${ }^{127}$ R. M. Harris, ${ }^{127}$ J. Hirschauer,${ }^{127}$ B. Hooberman, ${ }^{127}$ S. Jindariani, ${ }^{127}$ M. Johnson, ${ }^{127}$ U. Joshi, ${ }^{127}$ B. Klima, ${ }^{127}$ S. Kunori, ${ }^{127}$ S. Kwan, ${ }^{127}$ C. Leonidopoulos, ${ }^{127, b b b}$ J. Linacre, ${ }^{127}$ D. Lincoln, ${ }^{127}$ R. Lipton, ${ }^{127}$ J. Lykken, ${ }^{127}$ K. Maeshima, ${ }^{127}$ J. M. Marraffino, ${ }^{127}$ S. Maruyama, ${ }^{127}$ D. Mason, ${ }^{127}$ P. McBride,${ }^{127}$ K. Mishra, ${ }^{127}$ S. Mrenna, ${ }^{127}$ Y. Musienko, ${ }^{127, \mathrm{ccc}}$ C. Newman-Holmes, ${ }^{127}$ V. O’Dell, ${ }^{127}$ O. Prokofyev, ${ }^{127}$ E. Sexton-Kennedy, ${ }^{127}$ S. Sharma, ${ }^{127}$ W. J. Spalding, ${ }^{127}$ L. Spiegel, ${ }^{127}$ L. Taylor, ${ }^{127}$ S. Tkaczyk, ${ }^{127}$ N. V. Tran, ${ }^{127}$ L. Uplegger,,${ }^{127}$ E. W. Vaandering, ${ }^{127}$ R. Vidal,${ }^{127}$ J. Whitmore, ${ }^{127}$ W. Wu,${ }^{127}$ F. Yang, ${ }^{127}$ J. C. Yun, ${ }^{127}$ D. Acosta, ${ }^{128}$ P. Avery, ${ }^{128}$ D. Bourilkov, ${ }^{128}$ M. Chen, ${ }^{128}$ T. Cheng, ${ }^{128}$ S. Das, ${ }^{128}$ M. De Gruttola ${ }^{128}$ G. P. Di Giovanni, ${ }^{128}$ D. Dobur, ${ }^{128}$ A. Drozdetskiy, ${ }^{128}$ R. D. Field, ${ }^{128}$ M. Fisher, ${ }^{128}$ Y. Fu, ${ }^{128}$ I. K. Furic, ${ }^{128}$ J. Gartner, ${ }^{128}$ J. Hugon, ${ }^{128}$ B. Kim, ${ }^{128}$ J. Konigsberg, ${ }^{128}$ A. Korytov, ${ }^{128}$ A. Kropivnitskaya, ${ }^{128}$ T. Kypreos, ${ }^{128}$ J. F. Low, ${ }^{128}$ K. Matchev, ${ }^{128}$ P. Milenovic, ${ }^{128, \text { ddd }}$ G. Mitselmakher,${ }^{128}$ L. Muniz, ${ }^{128}$ M. Park, ${ }^{128}$ R. Remington, ${ }^{128}$ A. Rinkevicius, ${ }^{128}$ P. Sellers, ${ }^{128}$ N. Skhirtladze, ${ }^{128}$ M. Snowball, ${ }^{128}$ J. Yelton, ${ }^{128}$ M. Zakaria, ${ }^{128}$ V. Gaultney, ${ }^{129}$ S. Hewamanage,${ }^{129}$ L. M. Lebolo, ${ }^{129}$ S. Linn, ${ }^{129}$ P. Markowitz, ${ }^{129}$ G. Martinez, ${ }^{129}$ J.L. Rodriguez, ${ }^{129}$ T. Adams, ${ }^{130}$ A. Askew, ${ }^{130}$ J. Bochenek, ${ }^{130}$ J. Chen, ${ }^{130}$ B. Diamond, ${ }^{130}$ S. V. Gleyzer, ${ }^{130}$ J. Haas, ${ }^{130}$ S. Hagopian, ${ }^{130}$ V. Hagopian, ${ }^{130}$ M. Jenkins, ${ }^{130}$ K. F. Johnson, ${ }^{130}$ H. Prosper, ${ }^{130}$ V. Veeraraghavan, ${ }^{130}$ M. Weinberg, ${ }^{130}$ M. M. Baarmand, ${ }^{131}$ B. Dorney,${ }^{131}$ M. Hohlmann, ${ }^{131}$ H. Kalakhety, ${ }^{131}$ I. Vodopiyanov, ${ }^{131}$ F. Yumiceva, ${ }^{131}$ M. R. Adams, ${ }^{132}$ I. M. Anghel, ${ }^{132}$ L. Apanasevich ${ }^{132}$ Y. Bai, ${ }^{132}$ V. E. Bazterra, ${ }^{132}$ R. R. Betts, ${ }^{132}$ I. Bucinskaite, ${ }^{132}$ J. Callner, ${ }^{132}$ R. Cavanaugh, ${ }^{132}$ O. Evdokimov, ${ }^{132}$ L. Gauthier, ${ }^{132}$ C. E. Gerber, ${ }^{132}$ D. J. Hofman, ${ }^{132}$ S. Khalatyan, ${ }^{132}$ F. Lacroix, ${ }^{132}$ C. O’Brien, ${ }^{132}$ C. Silkworth, ${ }^{132}$ D. Strom, ${ }^{132}$ P. Turner, ${ }^{132}$ N. Varelas, ${ }^{132}$ U. Akgun, ${ }^{133}$ E. A. Albayrak, ${ }^{133}$ B. Bilki, ${ }^{133, e e e}$ W. Clarida, ${ }^{133}$ F. Duru, ${ }^{133}$ S. Griffiths, ${ }^{133}$ J.-P. Merlo, ${ }^{133}$ H. Mermerkaya, ${ }^{133, f f f}$ A. Mestvirishvili, ${ }^{133}$ A. Moeller, ${ }^{133}$ J. Nachtman, ${ }^{133}$ C. R. Newsom,${ }^{133}$ E. Norbeck, ${ }^{133}$ Y. Onel, ${ }^{133}$ F. Ozok, ${ }^{133, g g g}$ S. Sen, ${ }^{133}$ P. Tan, ${ }^{133}$ E. Tiras, ${ }^{133}$ J. Wetzel, ${ }^{133}$ T. Yetkin, ${ }^{133}$ K. Yi, ${ }^{133}$ B. A. Barnett, ${ }^{134}$ B. Blumenfeld, ${ }^{134}$ S. Bolognesi, ${ }^{134}$ D. Fehling, ${ }^{134}$ G. Giurgiu, ${ }^{134}$ A. V. Gritsan, ${ }^{134}$ Z. J. Guo, ${ }^{134}$ G. Hu, ${ }^{134}$ P. Maksimovic, ${ }^{134}$ M. Swartz, ${ }^{134}$ A. Whitbeck, ${ }^{134}$ P. Baringer, ${ }^{135}$ A. Bean, ${ }^{135}$ G. Benelli, ${ }^{135}$ R. P. Kenny III, ${ }^{135}$ M. Murray, ${ }^{135}$ D. Noonan, ${ }^{135}$ S. Sanders, ${ }^{135}$ R. Stringer, ${ }^{135}$ G. Tinti, ${ }^{135}$ J. S. Wood, ${ }^{135}$ A. F. Barfuss, ${ }^{136}$ T. Bolton, ${ }^{136}$ I. Chakaberia, ${ }^{136}$ A. Ivanov, ${ }^{136}$ S. Khalil, ${ }^{136}$ M. Makouski, ${ }^{136}$ Y. Maravin, ${ }^{136}$ S. Shrestha, ${ }^{136}$ I. Svintradze, ${ }^{136}$ J. Gronberg,,${ }^{137}$ D. Lange, ${ }^{137}$ F. Rebassoo, ${ }^{137}$ D. Wright, ${ }^{137}$ A. Baden, ${ }^{138}$ B. Calvert, ${ }^{138}$ S. C. Eno, ${ }^{138}$ J. A. Gomez, ${ }^{138}$ N. J. Hadley, ${ }^{138}$ R. G. Kellogg, ${ }^{138}$ M. Kirn, ${ }^{138}$ T. Kolberg, ${ }^{138}$ Y. Lu, ${ }^{138}$ M. Marionneau, ${ }^{138}$ A. C. Mignerey, ${ }^{138}$ K. Pedro, ${ }^{138}$ A. Skuja, ${ }^{138}$ J. Temple, ${ }^{138}$ M. B. Tonjes,${ }^{138}$ S. C. Tonwar, ${ }^{138}$ A. Apyan, ${ }^{139}$ G. Bauer, ${ }^{139}$ J. Bendavid, ${ }^{139}$ W. Busza, ${ }^{139}$ E. Butz, ${ }^{139}$ I. A. Cali, ${ }^{139}$ M. Chan, ${ }^{139}$ V. Dutta, ${ }^{139}$ G. Gomez Ceballos, ${ }^{139}$ M. Goncharov, ${ }^{139}$ Y. Kim, ${ }^{139}$ M. Klute, ${ }^{139}$ K. Krajczar, ${ }^{139}$,hhh A. Levin, ${ }^{139}$ P. D. Luckey, ${ }^{139}$ T. Ma,${ }^{139}$ S. Nahn, ${ }^{139}$ C. Paus,${ }^{139}$ D. Ralph, ${ }^{139}$ C. Roland,${ }^{139}$ G. Roland,${ }^{139}$ M. Rudolph,${ }^{139}$ G. S. F. Stephans, ${ }^{139}$ F. Stöckli, ${ }^{139}$ K. Sumorok, ${ }^{139}$ K. Sung, ${ }^{139}$ D. Velicanu, ${ }^{139}$ E. A. Wenger, ${ }^{139}$ R. Wolf, ${ }^{139}$ B. Wyslouch, ${ }^{139}$ M. Yang, ${ }^{139}$ Y. Yilmaz, ${ }^{139}$ A. S. Yoon, ${ }^{139}$ M. Zanetti, ${ }^{139}$ V. Zhukova, ${ }^{139}$ S. I. Cooper, ${ }^{140}$ B. Dahmes, ${ }^{140}$ A. De Benedetti, ${ }^{140}$ G. Franzoni,,${ }^{140}$ A. Gude, ${ }^{140}$ S. C. Kao, ${ }^{140}$ K. Klapoetke,${ }^{140}$ Y. Kubota, ${ }^{140}$ J. Mans, ${ }^{140}$ N. Pastika, ${ }^{140}$ R. Rusack, ${ }^{140}$ M. Sasseville, ${ }^{140}$ A. Singovsky, ${ }^{140}$ N. Tambe,${ }^{140}$ J. Turkewitz, ${ }^{140}$ L. M. Cremaldi, ${ }^{141}$ R. Kroeger, ${ }^{141}$ L. Perera, ${ }^{141}$ R. Rahmat, ${ }^{141}$ D. A. Sanders, ${ }^{141}$ E. Avdeeva, ${ }^{142}$ K. Bloom, ${ }^{142}$ S. Bose, ${ }^{142}$ D. R. Claes, ${ }^{142}$ A. Dominguez, ${ }^{142}$ M. Eads, ${ }^{142}$ J. Keller, ${ }^{142}$ I. Kravchenko, ${ }^{142}$ J. Lazo-Flores,${ }^{142}$ S. Malik, ${ }^{142}$ G. R. Snow, ${ }^{142}$ A. Godshalk, ${ }^{143}$ I. Iashvili, ${ }^{143}$ S. Jain, ${ }^{143}$ A. Kharchilava, ${ }^{143}$ A. Kumar, ${ }^{143}$

S. Rappoccio, ${ }^{143}$ G. Alverson, ${ }^{144}$ E. Barberis, ${ }^{144}$ D. Baumgartel, ${ }^{144}$ M. Chasco, ${ }^{144}$ J. Haley, ${ }^{144}$ D. Nash,${ }^{144}$ T. Orimoto, ${ }^{144}$ D. Trocino, ${ }^{144}$ D. Wood,${ }^{144}$ J. Zhang, ${ }^{144}$ A. Anastassov, ${ }^{145}$ K. A. Hahn, ${ }^{145}$ A. Kubik, ${ }^{145}$ L. Lusito, ${ }^{145}$ N. Mucia, ${ }^{145}$ N. Odell, ${ }^{145}$ R. A. Ofierzynski, ${ }^{145}$ B. Pollack, ${ }^{145}$ A. Pozdnyakov, ${ }^{145}$ M. Schmitt, ${ }^{145}$ S. Stoynev, ${ }^{145}$ 
M. Velasco, ${ }^{145}$ S. Won, ${ }^{145}$ L. Antonelli, ${ }^{146}$ D. Berry, ${ }^{146}$ A. Brinkerhoff, ${ }^{146}$ K. M. Chan, ${ }^{146}$ M. Hildreth, ${ }^{146}$ C. Jessop, ${ }^{146}$ D. J. Karmgard, ${ }^{146}$ J. Kolb, ${ }^{146}$ K. Lannon, ${ }^{146}$ W. Luo, ${ }^{146}$ S. Lynch,${ }^{146}$ N. Marinelli, ${ }^{146}$ D. M. Morse, ${ }^{146}$ T. Pearson, ${ }^{146}$ M. Planer, ${ }^{146}$ R. Ruchti, ${ }^{146}$ J. Slaunwhite, ${ }^{146}$ N. Valls,${ }^{146}$ M. Wayne, ${ }^{146}$ M. Wolf, ${ }^{146}$ B. Bylsma,${ }^{147}$ L. S. Durkin, ${ }^{147}$ C. Hill, ${ }^{147}$ R. Hughes, ${ }^{147}$ K. Kotov, ${ }^{147}$ T. Y. Ling, ${ }^{147}$ D. Puigh, ${ }^{147}$ M. Rodenburg, ${ }^{147}$ C. Vuosalo, ${ }^{147}$ G. Williams, ${ }^{147}$ B. L. Winer, ${ }^{147}$ E. Berry, ${ }^{148}$ P. Elmer, ${ }^{148}$ V. Halyo, ${ }^{148}$ P. Hebda, ${ }^{148}$ J. Hegeman, ${ }^{148}$ A. Hunt,${ }^{148}$ P. Jindal, ${ }^{148}$ S. A. Koay, ${ }^{148}$ D. Lopes Pegna, ${ }^{148}$ P. Lujan, ${ }^{148}$ D. Marlow, ${ }^{148}$ T. Medvedeva, ${ }^{148}$ M. Mooney, ${ }^{148}$ J. Olsen, ${ }^{148}$ P. Piroué, ${ }^{148}$ X. Quan, ${ }^{148}$ A. Raval, ${ }^{148}$ H. Saka, ${ }^{148}$ D. Stickland, ${ }^{148}$ C. Tully, ${ }^{148}$ J. S. Werner, ${ }^{148}$ A. Zuranski, ${ }^{148}$ E. Brownson, ${ }^{149}$ A. Lopez, ${ }^{149}$ H. Mendez, ${ }^{149}$ J. E. Ramirez Vargas, ${ }^{149}$ E. Alagoz, ${ }^{150}$ V. E. Barnes, ${ }^{150}$ D. Benedetti, ${ }^{150}$ G. Bolla, ${ }^{150}$ D. Bortoletto, ${ }^{150}$ M. De Mattia, ${ }^{150}$ A. Everett, ${ }^{150}$ Z. Hu, ${ }^{150}$ M. Jones, ${ }^{150}$ O. Koybasi, ${ }^{150}$ M. Kress, ${ }^{150}$ A. T. Laasanen, ${ }^{150}$ N. Leonardo,${ }^{150}$ V. Maroussov, ${ }^{150}$ P. Merkel, ${ }^{150}$ D. H. Miller, ${ }^{150}$ N. Neumeister, ${ }^{150}$ I. Shipsey, ${ }^{150}$ D. Silvers, ${ }^{150}$ A. Svyatkovskiy, ${ }^{150}$ M. Vidal Marono, ${ }^{150}$ H. D. Yoo, ${ }^{150}$ J. Zablocki, ${ }^{150}$ Y. Zheng, ${ }^{150}$ S. Guragain, ${ }^{151}$ N. Parashar, ${ }^{151}$ A. Adair,${ }^{152}$ B. Akgun, ${ }^{152}$ C. Boulahouache, ${ }^{152}$ K. M. Ecklund,,${ }^{152}$ F. J. M. Geurts, ${ }^{152}$ W. Li, ${ }^{152}$ B. P. Padley, ${ }^{152}$ R. Redjimi, ${ }^{152}$ J. Roberts, ${ }^{152}$ J. Zabel, ${ }^{152}$ B. Betchart, ${ }^{153}$ A. Bodek, ${ }^{153}$ Y. S. Chung, ${ }^{153}$ R. Covarelli, ${ }^{153}$ P. de Barbaro, ${ }^{153}$ R. Demina, ${ }^{153}$ Y. Eshaq, ${ }^{153}$ T. Ferbel, ${ }^{153}$ A. Garcia-Bellido, ${ }^{153}$ P. Goldenzweig, ${ }^{153}$ J. Han, ${ }^{153}$ A. Harel, ${ }^{153}$ D. C. Miner, ${ }^{153}$ D. Vishnevskiy, ${ }^{153}$ M. Zielinski, ${ }^{153}$ A. Bhatti, ${ }^{154}$ R. Ciesielski, ${ }^{154}$ L. Demortier, ${ }^{154}$ K. Goulianos, ${ }^{154}$ G. Lungu, ${ }^{154}$ S. Malik, ${ }^{154}$ C. Mesropian, ${ }^{154}$ S. Arora, ${ }^{155}$ A. Barker, ${ }^{155}$ J. P. Chou, ${ }^{155}$ C. Contreras-Campana, ${ }^{155}$ E. Contreras-Campana, ${ }^{155}$ D. Duggan, ${ }^{155}$ D. Ferencek, ${ }^{155}$ Y. Gershtein, ${ }^{155}$ R. Gray, ${ }^{155}$ E. Halkiadakis, ${ }^{155}$ D. Hidas, ${ }^{155}$ A. Lath, ${ }^{155}$ S. Panwalkar, ${ }^{155}$ M. Park, ${ }^{155}$ R. Patel, ${ }^{155}$ V. Rekovic,${ }^{155}$ J. Robles, ${ }^{155}$ K. Rose, ${ }^{155}$ S. Salur, ${ }^{155}$ S. Schnetzer,${ }^{155}$ C. Seitz, ${ }^{155}$ S. Somalwar, ${ }^{155}$ R. Stone,${ }^{155}$ S. Thomas,${ }^{155}$ M. Walker, ${ }^{155}$ G. Cerizza, ${ }^{156}$ M. Hollingsworth, ${ }^{156}$ S. Spanier, ${ }^{156}$ Z. C. Yang, ${ }^{156}$ A. York,,${ }^{156}$ R. Eusebi, ${ }^{157}$ W. Flanagan, ${ }^{157}$ J. Gilmore, ${ }^{157}$ T. Kamon, ${ }^{157, \text { iii }}$ V. Khotilovich, ${ }^{157}$ R. Montalvo, ${ }^{157}$ I. Osipenkov, ${ }^{157}$ Y. Pakhotin, ${ }^{157}$ A. Perloff, ${ }^{157}$ J. Roe, ${ }^{157}$ A. Safonov, ${ }^{157}$ T. Sakuma, ${ }^{157}$ S. Sengupta, ${ }^{157}$ I. Suarez, ${ }^{157}$ A. Tatarinov, ${ }^{157}$ D. Toback, ${ }^{157}$ N. Akchurin, ${ }^{158}$ J. Damgov, ${ }^{158}$ C. Dragoiu, ${ }^{158}$ P. R. Dudero, ${ }^{158}$ C. Jeong, ${ }^{158}$ K. Kovitanggoon, ${ }^{158}$ S. W. Lee ${ }^{158}$

T. Libeiro, ${ }^{158}$ I. Volobouev, ${ }^{158}$ E. Appelt, ${ }^{159}$ A. G. Delannoy, ${ }^{159}$ C. Florez, ${ }^{159}$ S. Greene, ${ }^{159}$ A. Gurrola, ${ }^{159}$ W. Johns, ${ }^{159}$ P. Kurt, ${ }^{159}$ C. Maguire, ${ }^{159}$ A. Melo, ${ }^{159}$ M. Sharma, ${ }^{159}$ P. Sheldon, ${ }^{159}$ B. Snook, ${ }^{159}$ S. Tuo, ${ }^{159}$ J. Velkovska, ${ }^{159}$ M. W. Arenton, ${ }^{160}$ M. Balazs, ${ }^{160}$ S. Boutle,${ }^{160}$ B. Cox, ${ }^{160}$ B. Francis, ${ }^{160}$ J. Goodell, ${ }^{160}$ R. Hirosky, ${ }^{160}$ A. Ledovskoy, ${ }^{160}$ C. Lin, ${ }^{160}$ C. Neu, ${ }^{160}$ J. Wood,${ }^{160}$ S. Gollapinni, ${ }^{161}$ R. Harr, ${ }^{161}$ P. E. Karchin,${ }^{161}$

C. Kottachchi Kankanamge Don, ${ }^{161}$ P. Lamichhane, ${ }^{161}$ A. Sakharov, ${ }^{161}$ M. Anderson, ${ }^{162}$ D. A. Belknap, ${ }^{162}$ L. Borrello, ${ }^{162}$ D. Carlsmith, ${ }^{162}$ M. Cepeda, ${ }^{162}$ S. Dasu, ${ }^{162}$ E. Friis, ${ }^{162}$ L. Gray, ${ }^{162}$ K. S. Grogg,,${ }^{162}$ M. Grothe,${ }^{162}$ R. Hall-Wilton, ${ }^{162}$ M. Herndon, ${ }^{162}$ A. Hervé, ${ }^{162}$ P. Klabbers, ${ }^{162}$ J. Klukas, ${ }^{162}$ A. Lanaro, ${ }^{162}$ C. Lazaridis, ${ }^{162}$ R. Loveless, ${ }^{162}$ A. Mohapatra, ${ }^{162}$ I. Ojalvo, ${ }^{162}$ F. Palmonari, ${ }^{162}$ G. A. Pierro, ${ }^{162}$ I. Ross, ${ }^{162}$ A. Savin, ${ }^{162}$ W. H. Smith, ${ }^{162}$ and J. Swanson ${ }^{162}$

\title{
(CMS Collaboration)
}

\author{
${ }^{1}$ Yerevan Physics Institute, Yerevan, Armenia \\ ${ }^{2}$ Institut für Hochenergiephysik der OeAW, Wien, Austria \\ ${ }^{3}$ National Centre for Particle and High Energy Physics, Minsk, Belarus \\ ${ }^{4}$ Universiteit Antwerpen, Antwerpen, Belgium \\ ${ }^{5}$ Vrije Universiteit Brussel, Brussel, Belgium \\ ${ }^{6}$ Université Libre de Bruxelles, Bruxelles, Belgium \\ ${ }^{7}$ Ghent University, Ghent, Belgium \\ ${ }^{8}$ Université Catholique de Louvain, Louvain-la-Neuve, Belgium \\ ${ }^{9}$ Université de Mons, Mons, Belgium \\ ${ }^{10}$ Centro Brasileiro de Pesquisas Fisicas, Rio de Janeiro, Brazil \\ ${ }^{11}$ Universidade do Estado do Rio de Janeiro, Rio de Janeiro, Brazil \\ ${ }^{12 a}$ Universidade Estadual Paulista, São Paulo, Brazil \\ ${ }^{12 \mathrm{~b}}$ Universidade Federal do ABC, São Paulo, Brazil \\ ${ }^{13}$ Institute for Nuclear Research and Nuclear Energy, Sofia, Bulgaria \\ ${ }^{14}$ University of Sofia, Sofia, Bulgaria \\ ${ }^{15}$ Institute of High Energy Physics, Beijing, China \\ ${ }^{16}$ State Key Laboratory of Nuclear Physics and Technology, Peking University, Beijing, China \\ ${ }^{17}$ Universidad de Los Andes, Bogota, Colombia \\ ${ }^{18}$ Technical University of Split, Split, Croatia
}






${ }^{31}$ Centre de Calcul de l'Institut National de Physique Nucleaire et de Physique des Particules, CNRS/IN2P3, Villeurbanne, France

${ }^{32}$ Université de Lyon, Université Claude Bernard Lyon 1, CNRS-IN2P3, Institut de Physique Nucléaire de Lyon, Villeurbanne, France

${ }^{33}$ Institute of High Energy Physics and Informatization, Tbilisi State University, Tbilisi, Georgia

${ }^{34}$ RWTH Aachen University, I. Physikalisches Institut, Aachen, Germany

${ }^{35}$ RWTH Aachen University, III. Physikalisches Institut A, Aachen, Germany

${ }^{36}$ RWTH Aachen University, III. Physikalisches Institut B, Aachen, Germany

${ }^{37}$ Deutsches Elektronen-Synchrotron, Hamburg, Germany

${ }^{38}$ University of Hamburg, Hamburg, Germany

${ }^{39}$ Institut für Experimentelle Kernphysik, Karlsruhe, Germany

${ }^{40}$ Institute of Nuclear Physics “Demokritos”, Aghia Paraskevi, Greece

${ }^{41}$ University of Athens, Athens, Greece

${ }^{42}$ University of Ioánnina, Ioánnina, Greece

${ }^{43}$ KFKI Research Institute for Particle and Nuclear Physics, Budapest, Hungary

${ }^{44}$ Institute of Nuclear Research ATOMKI, Debrecen, Hungary

${ }^{45}$ University of Debrecen, Debrecen, Hungary

${ }^{46}$ Panjab University, Chandigarh, India

${ }^{47}$ University of Delhi, Delhi, India

${ }^{48}$ Saha Institute of Nuclear Physics, Kolkata, India

${ }^{49}$ Bhabha Atomic Research Centre, Mumbai, India

${ }^{50}$ Tata Institute of Fundamental Research-EHEP, Mumbai, India

${ }^{51}$ Tata Institute of Fundamental Research-HECR, Mumbai, India

${ }^{52}$ Institute for Research in Fundamental Sciences (IPM), Tehran, Iran

${ }^{53 a}$ INFN Sezione di Bari, Bari, Italy

${ }^{53 \mathrm{~b}}$ Università di Bari, Bari, Italy

${ }^{53 c}$ Politecnico di Bari, Bari, Italy

${ }^{54 a}$ INFN Sezione di Bologna, Bologna, Italy

${ }^{54 \mathrm{~b}}$ Università di Bologna, Bologna, Italy

${ }^{55}$ INFN Sezione di Catania, Catania, Italy

${ }^{55 b}$ Università di Catania, Catania, Italy

${ }^{56 a}$ INFN Sezione di Firenze, Firenze, Italy

${ }^{56 \mathrm{~b}}$ Università di Firenze, Firenze, Italy

${ }^{57}$ INFN Laboratori Nazionali di Frascati, Frascati, Italy

${ }^{58 a}$ INFN Sezione di Genova, Genova, Italy

${ }^{58 \mathrm{~b}}$ Università di Genova, Genova, Italy

${ }^{59}$ a INFN Sezione di Milano-Bicocca, Milano, Italy

${ }^{59 b}$ Università di Milano-Bicocca, Milano, Italy

${ }^{60 a}$ INFN Sezione di Napoli, Napoli, Italy

${ }^{60 \mathrm{~b}}$ Università di Napoli 'Federico II', Napoli, Italy

${ }^{60 c}$ Università della Basilicata (Potenza), Napoli, Italy

${ }^{60 \mathrm{~d}}$ Università G. Marconi (Roma), Napoli, Italy

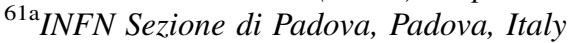

${ }^{61 \mathrm{~b}}$ Università di Padova, Padova, Italy

${ }^{61 \mathrm{c}}$ Università di Trento (Trento), Padova, Italy

${ }^{62 \mathrm{a}}$ INFN Sezione di Pavia, Pavia, Italy

${ }^{62 \mathrm{~b}}$ Università di Pavia, Pavia, Italy

${ }^{63 a}$ INFN Sezione di Perugia, Perugia, Italy

${ }^{63 \mathrm{~b}}$ Università di Perugia, Perugia, Italy 
${ }^{64 a}$ INFN Sezione di Pisa, Pisa, Italy

${ }^{64 \mathrm{~b}}$ Università di Pisa, Pisa, Italy

${ }^{64 \mathrm{c}}$ Scuola Normale Superiore di Pisa, Pisa, Italy

${ }^{65 a}$ INFN Sezione di Roma, Roma, Italy

${ }^{65 \mathrm{~b}}$ Università di Roma, Roma, Italy

${ }^{66 \mathrm{a}}$ INFN Sezione di Torino, Torino, Italy

${ }^{66 \mathrm{~b}}$ Università di Torino, Torino, Italy

${ }^{66 c}$ Università del Piemonte Orientale (Novara), Torino, Italy

${ }^{67 \mathrm{a}}$ INFN Sezione di Trieste, Trieste, Italy

${ }^{67 \mathrm{~b}}$ Università di Trieste, Trieste, Italy

${ }^{68}$ Kangwon National University, Chunchon, Korea

${ }^{69}$ Kyungpook National University, Daegu, Korea

${ }^{70}$ Chonnam National University, Institute for Universe and Elementary Particles, Kwangju, Korea

${ }^{71}$ Korea University, Seoul, Korea

${ }^{72}$ University of Seoul, Seoul, Korea

${ }^{73}$ Sungkyunkwan University, Suwon, Korea

${ }^{74}$ Vilnius University, Vilnius, Lithuania

${ }^{75}$ Centro de Investigacion y de Estudios Avanzados del IPN, Mexico City, Mexico

${ }^{76}$ Universidad Iberoamericana, Mexico City, Mexico

${ }^{77}$ Benemerita Universidad Autonoma de Puebla, Puebla, Mexico

${ }^{78}$ Universidad Autónoma de San Luis Potosí, San Luis Potosí, Mexico

${ }^{79}$ University of Auckland, Auckland, New Zealand

${ }^{80}$ University of Canterbury, Christchurch, New Zealand

${ }^{81}$ National Centre for Physics, Quaid-I-Azam University, Islamabad, Pakistan

${ }^{82}$ National Centre for Nuclear Research, Swierk, Poland

${ }^{83}$ Institute of Experimental Physics, Faculty of Physics, University of Warsaw, Warsaw, Poland

${ }^{84}$ Laboratório de Instrumentação e Física Experimental de Partículas, Lisboa, Portugal

${ }^{85}$ Joint Institute for Nuclear Research, Dubna, Russia

${ }^{86}$ Petersburg Nuclear Physics Institute, Gatchina (St. Petersburg), Russia

${ }^{87}$ Institute for Nuclear Research, Moscow, Russia

${ }^{88}$ Institute for Theoretical and Experimental Physics, Moscow, Russia

${ }^{89}$ Moscow State University, Moscow, Russia

${ }^{90}$ P.N. Lebedev Physical Institute, Moscow, Russia

${ }^{91}$ State Research Center of Russian Federation, Institute for High Energy Physics, Protvino, Russia

${ }^{92}$ University of Belgrade, Faculty of Physics and Vinca Institute of Nuclear Sciences, Belgrade, Serbia

${ }^{93}$ Centro de Investigaciones Energéticas Medioambientales y Tecnológicas (CIEMAT), Madrid, Spain

${ }^{94}$ Universidad Autónoma de Madrid, Madrid, Spain

${ }^{95}$ Universidad de Oviedo, Oviedo, Spain

${ }^{96}$ Instituto de Física de Cantabria (IFCA), CSIC-Universidad de Cantabria, Santander, Spain

${ }^{97}$ CERN, European Organization for Nuclear Research, Geneva, Switzerland

${ }^{98}$ Paul Scherrer Institut, Villigen, Switzerland

${ }^{99}$ Institute for Particle Physics, ETH Zurich, Zurich, Switzerland

${ }^{100}$ Universität Zürich, Zurich, Switzerland

${ }^{101}$ National Central University, Chung-Li, Taiwan

${ }^{102}$ National Taiwan University (NTU), Taipei, Taiwan

${ }^{103}$ Chulalongkorn University, Bangkok, Thailand

${ }^{104}$ Cukurova University, Adana, Turkey

${ }^{105}$ Middle East Technical University, Physics Department, Ankara, Turkey

${ }^{106}$ Bogazici University, Istanbul, Turkey

${ }^{107}$ Istanbul Technical University, Istanbul, Turkey

${ }^{108}$ National Scientific Center, Kharkov Institute of Physics and Technology, Kharkov, Ukraine

${ }^{109}$ University of Bristol, Bristol, United Kingdom

${ }^{110}$ Rutherford Appleton Laboratory, Didcot, United Kingdom

${ }^{111}$ Imperial College, London, United Kingdom

${ }^{112}$ Brunel University, Uxbridge, United Kingdom

${ }^{113}$ Baylor University, Waco, Texas, USA

${ }^{114}$ The University of Alabama, Tuscaloosa, Alabama, USA

${ }^{115}$ Boston University, Boston, Massachusetts, USA

${ }^{116}$ Brown University, Providence, Rhode Island, USA

${ }^{117}$ University of California, Davis, Davis, California, USA

${ }^{118}$ University of California, Los Angeles, California, USA 
${ }^{119}$ University of California, Riverside, Riverside, California, USA

${ }^{120}$ University of California, San Diego, La Jolla, California, USA

${ }^{121}$ University of California, Santa Barbara, Santa Barbara, California, USA

${ }^{122}$ California Institute of Technology, Pasadena, California, USA

${ }^{123}$ Carnegie Mellon University, Pittsburgh, Pennsylvania, USA

${ }^{124}$ University of Colorado at Boulder, Boulder, Colorado, USA

${ }^{125}$ Cornell University, Ithaca, New York, USA

${ }^{126}$ Fairfield University, Fairfield, Connecticut, USA

${ }^{127}$ Fermi National Accelerator Laboratory, Batavia, Illinois, USA

${ }^{128}$ University of Florida, Gainesville, Florida, USA

${ }^{129}$ Florida International University, Miami, Florida, USA

${ }^{130}$ Florida State University, Tallahassee, Florida, USA

${ }^{131}$ Florida Institute of Technology, Melbourne, Florida, USA

${ }^{132}$ University of Illinois at Chicago (UIC), Chicago, Illinois, USA

${ }^{133}$ The University of Iowa, Iowa City, Iowa, USA

${ }^{134}$ Johns Hopkins University, Baltimore, Maryland, USA

${ }^{135}$ The University of Kansas, Lawrence, Kansas, USA

${ }^{136}$ Kansas State University, Manhattan, Kansas, USA

${ }^{137}$ Lawrence Livermore National Laboratory, Livermore, California, USA

${ }^{138}$ University of Maryland, College Park, Maryland, USA

${ }^{139}$ Massachusetts Institute of Technology, Cambridge, Massachusetts, USA

${ }^{140}$ University of Minnesota, Minneapolis, Minnesota, USA

${ }^{141}$ University of Mississippi, Oxford, Mississippi, USA

${ }^{142}$ University of Nebraska-Lincoln, Lincoln, Nebraska, USA

${ }^{143}$ State University of New York at Buffalo, Buffalo, New York, USA

${ }^{144}$ Northeastern University, Boston, Massachusetts, USA

${ }^{145}$ Northwestern University, Evanston, Illinois, USA

${ }^{146}$ University of Notre Dame, Notre Dame, Indiana, USA

${ }^{147}$ The Ohio State University, Columbus, Ohio, USA

${ }^{148}$ Princeton University, Princeton, New Jersey, USA

${ }^{149}$ University of Puerto Rico, Mayaguez, Puerto Rico

${ }^{150}$ Purdue University, West Lafayette, Indiana, USA

${ }^{151}$ Purdue University Calumet, Hammond, Indiana, USA

${ }^{152}$ Rice University, Houston, Texas, USA

${ }^{153}$ University of Rochester, Rochester, New York, USA

${ }^{154}$ The Rockefeller University, New York, New York, USA

${ }^{155}$ Rutgers, the State University of New Jersey, Piscataway, New Jersey, USA

${ }^{156}$ University of Tennessee, Knoxville, Tennessee, USA

${ }^{157}$ Texas A\&M University, College Station, Texas, USA

${ }^{158}$ Texas Tech University, Lubbock, Texas, USA

${ }^{159}$ Vanderbilt University, Nashville, Tennessee, USA

${ }^{160}$ University of Virginia, Charlottesville, Virginia, USA

${ }^{161}$ Wayne State University, Detroit, Michigan, USA

${ }^{162}$ University of Wisconsin, Madison, Wisconsin, USA

${ }^{\mathrm{a}}$ Deceased.

${ }^{\mathrm{b}}$ Also at Vienna University of Technology, Vienna, Austria.

${ }^{\mathrm{c}}$ Also at CERN, European Organization for Nuclear Research, Geneva, Switzerland.

${ }^{\mathrm{d}}$ Also at National Institute of Chemical Physics and Biophysics, Tallinn, Estonia.

${ }^{\mathrm{e}}$ Also at California Institute of Technology, Pasadena, USA.

${ }^{\mathrm{f}}$ Also at Laboratoire Leprince-Ringuet, Ecole Polytechnique, IN2P3-CNRS, Palaiseau, France.

${ }^{\mathrm{g}}$ Also at Suez Canal University, Suez, Egypt.

${ }^{\mathrm{h}}$ Also at Zewail City of Science and Technology, Zewail, Egypt.

${ }^{\mathrm{i}}$ Also at Cairo University, Cairo, Egypt.

${ }^{\mathrm{j}}$ Also at Fayoum University, El-Fayoum, Egypt.

${ }^{\mathrm{k}}$ Also at Helwan University, Cairo, Egypt.

${ }^{1}$ Also at British University in Egypt, Cairo, Egypt.

${ }^{\mathrm{m}}$ Now at Ain Shams University, Cairo, Egypt.

${ }^{\mathrm{n}}$ Also at National Centre for Nuclear Research, Swierk, Poland. 
${ }^{\circ}$ Now at Université de Haute Alsace, Mulhouse, France.

${ }^{\mathrm{p}}$ Also at Joint Institute for Nuclear Research, Dubna, Russia.

${ }^{\mathrm{q}}$ Also at Moscow State University, Moscow, Russia.

${ }^{\mathrm{r}}$ Also at Brandenburg University of Technology, Cottbus, Germany.

${ }^{\mathrm{s}}$ Also at The University of Kansas, Lawrence, KS, USA.

${ }^{\mathrm{t}}$ Also at Institute of Nuclear Research ATOMKI, Debrecen, Hungary.

uAlso at Eötvös Loránd University, Budapest, Hungary.

${ }^{v}$ Also at Tata Institute of Fundamental Research-HECR, Mumbai, India.

${ }^{\mathrm{w}}$ Now at King Abdulaziz University, Jeddah, Saudi Arabia.

${ }^{\mathrm{x}}$ Also at University of Visva-Bharati, Santiniketan, India.

${ }^{\mathrm{y}}$ Also at Sharif University of Technology, Tehran, Iran.

${ }^{\mathrm{z}}$ Also at Isfahan University of Technology, Isfahan, Iran.

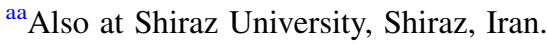

${ }^{\mathrm{bb}}$ Also at Plasma Physics Research Center, Science and Research Branch, Islamic Azad University, Tehran, Iran.

${ }^{c c}$ Also at Facoltà Ingegneria, Università di Roma, Roma, Italy.

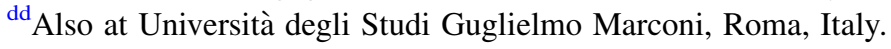

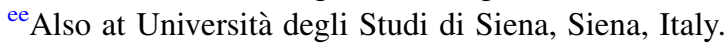

${ }^{\mathrm{ff}}$ Also at University of Bucharest, Faculty of Physics, Bucuresti-Magurele, Romania.

${ }^{g g}$ Also at Faculty of Physics of University of Belgrade, Belgrade, Serbia.

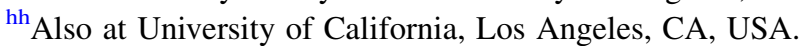

${ }^{\text {ii } A l s o ~ a t ~ S c u o l a ~ N o r m a l e ~ e ~ S e z i o n e ~ d e l l ' I N F N, ~ P i s a, ~ I t a l y . ~}$

${ }^{\mathrm{jj}}$ Also at INFN Sezione di Roma, Roma, Italy.

${ }^{\mathrm{kk}}$ Also at University of Athens, Athens, Greece.

${ }^{11}$ Also at Rutherford Appleton Laboratory, Didcot, United Kingdom.

${ }^{\mathrm{mm}}$ Also at Paul Scherrer Institut, Villigen, Switzerland.

${ }^{n n}$ Also at Institute for Theoretical and Experimental Physics, Moscow, Russia.

${ }^{\circ o}$ Also at Albert Einstein Center for Fundamental Physics, Bern, Switzerland.

${ }^{p p}$ Also at Gaziosmanpasa University, Tokat, Turkey.

${ }^{\mathrm{qq}}$ Also at Adiyaman University, Adiyaman, Turkey.

${ }^{\mathrm{rr}}$ Also at Izmir Institute of Technology, Izmir, Turkey

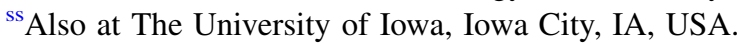

${ }^{\text {tt } A l s o ~ a t ~ M e r s i n ~ U n i v e r s i t y, ~ M e r s i n, ~ T u r k e y . ~}$



${ }^{\mathrm{vv}}$ Also at Kafkas University, Kars, Turkey.

${ }^{w w}$ Also at Suleyman Demirel University, Isparta, Turkey.

${ }^{\mathrm{xx}}$ Also at Ege University, Izmir, Turkey.

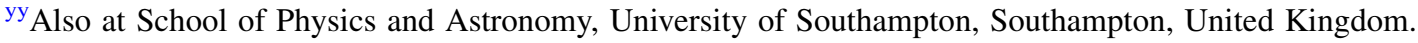

${ }^{z z}$ Also at INFN Sezione di Perugia, Università di Perugia, Perugia, Italy.

${ }^{\text {aaa }}$ Also at Utah Valley University, Orem, UT, USA.

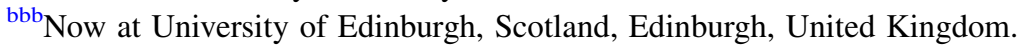

${ }^{c c c}$ Also at Institute for Nuclear Research, Moscow, Russia.

${ }^{\text {ddd }}$ Also at University of Belgrade, Faculty of Physics and Vinca Institute of Nuclear Sciences, Belgrade, Serbia.

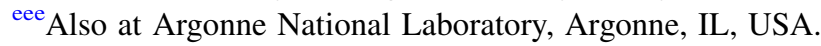

${ }^{\mathrm{fff}}$ Also at Erzincan University, Erzincan, Turkey.

${ }^{\mathrm{ggg}}$ Also at Mimar Sinan University, Istanbul, Istanbul, Turkey.

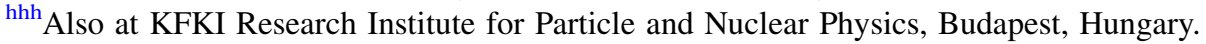

${ }^{i i i}$ Also at Kyungpook National University, Daegu, Korea. 